\title{
The role of brain size on mammalian population densities
}

Article

Accepted Version

Gonzalez-Suarez, M. ORCID: https://orcid.org/0000-00015069-8900, Gonzalez-Voyer, A., von Hardenber, A. and Santini, L. (2021) The role of brain size on mammalian population densities. Journal of Animal Ecology, 90 (3). pp. 653-661. ISSN 0021-8790 doi: https://doi.org/10.1111/13652656.13397 Available at https://centaur.reading.ac.uk/93807/

It is advisable to refer to the publisher's version if you intend to cite from the work. See Guidance on citing.

To link to this article DOI: http://dx.doi.org/10.1111/1365-2656.13397

Publisher: Wiley

All outputs in CentAUR are protected by Intellectual Property Rights law, including copyright law. Copyright and IPR is retained by the creators or other copyright holders. Terms and conditions for use of this material are defined in the End User Agreement.

\section{www.reading.ac.uk/centaur}

\section{CentAUR}

Central Archive at the University of Reading 
Reading's research outputs online 
Title: The role of brain size on mammalian population densities

Short title: Population density and brain size

Authors: Manuela González-Suárez ${ }^{1,}$, Alejandro Gonzalez-Voyer ${ }^{2}$, Achaz von Hardenberg ${ }^{3}$, Luca Santini ${ }^{4,5}$

\section{Affiliations:}

${ }^{1}$ Ecology and Evolutionary Biology, School of Biological Sciences, University of Reading, Whiteknights, Reading, RG6 6AS, UK

${ }^{2}$ Departamento de Ecología Evolutiva, Instituto de Ecología, Universidad Nacional Autónoma de México, Cd. México, 04510, México.

${ }^{3}$ Conservation Biology Research Group, Department of Biological Sciences, University of Chester, Chester, CH1 4BJ UK

${ }^{4}$ Department of Environmental Science, Institute for Wetland and Water Research, Faculty of Science, Radboud University, P.O. Box 9010, NL-6500 GL, Nijmegen, The Netherlands.

${ }^{5}$ National Research Council, Institute of Research on Terrestrial Ecosystems (CNR-IRET), Via Salaria km 29.300, 00015, Monterotondo (Rome), Italy

*corresponding author: manuela.gonzalez@ reading.ac.uk 


\section{Abstract}

1. The local abundance or population density of different organisms often varies widely. Understanding what determines this variation is an important, but not yet fully resolved question in ecology. Differences in population density are partly driven by variation in body size and diet among organisms. Here we propose the size of an organism' brain could be an additional, overlooked, driver of mammalian population densities.

2. We explore two possible contrasting mechanisms by which brain size, measured by its mass, could affect population density. First, because of the energetic demands of larger brains and their influence on life history, we predict mammals with larger relative brain masses would occur at lower population densities. Alternatively, larger brains are generally associated with a greater ability to exploit new resources, which would provide a competitive advantage leading to higher population densities among large-brained mammals.

3. We tested these predictions using phylogenetic path analysis, modelling hypothesized direct and indirect relationships between diet, body mass, brain mass and population density for 656 nonvolant terrestrial mammalian species. We analysed all data together and separately for marsupials and the four taxonomic orders with most species in the dataset (Carnivora, Cetartiodactyla, Primates, Rodentia).

4. For all species combined, a single model was supported showing lower population density associated with larger brains, larger bodies, and more specialized diets. The negative effect of brain mass was also supported for separate analyses in Primates and Carnivora. In other groups (Rodentia, Cetartiodactyla and marsupials) the relationship was less clear: supported models included a direct link from brain mass to population density but $95 \%$ confidence intervals of the path coefficients overlapped zero.

5. Results support our hypothesis that brain mass can explain variation in species' average population density, with large-brained species having greater area requirements, although the relationship may vary across taxonomic groups. Future research is needed to clarify whether the 
role of brain mass on population density varies as a function of environmental (e.g. environmental stability) and biotic conditions (e.g. level of competition).

Keywords: Body mass, Body size, Brain mass, Comparative methods, Diet, Mammalia, Phylogenetic path analysis, Population abundance. 


\section{Introduction}

Ecologists observed long ago that different organisms occupy natural environments at different population densities, yet what determines population density is still considered one of the unresolved problems in ecology (Dobson, Tilman \& Holt 2020). Earlier studies proposed variation in population density among species could be explained by a combination of species' energy requirements and the availability and accessibility of resources (Damuth 1981; Brown 1995). These proposed relationships led to a pursuit of general rules linking interspecific variation in population density with energy requirements (White, Ernest, Kerkhoff \& Enquist 2007). Because directly measuring energy requirements across numerous species is challenging, macroecological research has relied on proxies, primarily body mass and to a lesser extent diet composition, to test these hypotheses (Damuth 1981; Silva, Brimacombe \& Downing 2001). For example, the 'energy equivalence rule' proposes that population density decreases with body mass with a power exponent of -0.75 , which is the reciprocal of the rate by which individual metabolic rates increase with body size (Damuth 2007; White et al. 2007). This rule and its proposed exponent have been challenged on theoretical and empirical grounds (Blackburn \& Gaston 1999; Isaac, Storch \& Carbone 2013), with research showing that the power exponent varies across taxonomic groups (Isaac, Storch \& Carbone 2011; Pedersen, Faurby \& Svenning 2017). However, the fact that an average species' population density is associated with its average body mass remains largely supported (Isaac et al. 2011; Santini et al. 2018a). Everything else equal, a larger organism will require a larger area than a smaller organism to meet its energetic requirements. In addition, energy requirements can also depend on the composition of an organism's diet for two reasons. First, animals that consume less abundant and more scattered resources are expected to need larger areas to fulfil their requirements. Second, different diet items are associated with different availabilities, foraging costs, and energy assimilation efficiencies (Silva, Brown \& Downing 1997). For example, mammals with more nectivorous and more carnivorous diets have higher energy requirements (Nagy, Girard \& Brown 1999; Anderson \& Jetz 2005). 
In mammals, energetic requirements are also influenced by brain size (Mink, Blumenschine \& Adams 1981; Herculano-Houzel 2011). Empirical data shows that cerebral energy use increases more steeply with brain size than whole-body energy use does with increased body size (Karbowski 2007) such that species with larger brains have higher metabolic rates after controlling for allometric effects (Hofman 1983; Isler \& van Schaik 2006). Brains are also costly to develop, and species with larger brains generally have reduced reproductive output and longer development times, which result in slower potential population growth rates and lower abundance (Isler \& van Schaik 2009; Barton \& Capellini 2011; Gonzalez-Voyer, González-Suárez, Vilà \& Revilla 2016). Despite the extensive evidence that brain size influences energy requirements, the potential role of brain mass as a predictor of average population densities has remained largely unexplored, possibly due to its high correlation with body mass and reduced availability of brain size estimates. A recent study evaluating extinction risk among mammals suggested a possible direct negative relationship between brain size and population density (Gonzalez-Voyer et al. 2016). Yet, this link was not further explored, and that study did not account for other known drivers of population density such as diet composition. In addition to the energy effect, brain size could influence population density but show a positive relationship. Larger brains have been linked to greater cognitive ability and innovation capacity, which could provide a competitive advantage over other species, including greater ability to locate ephemeral food patches and use more diverse resources in an environment, and provide advantages in coping with new conditions (Visalberghi, Fragaszy \& Savage-Rumbaugh 1995; Sol, Timmermans \& Lefebvre 2002; Amiel, Tingley \& Shine 2011; Maklakov, Immler, Gonzalez-Voyer, Rönn \& Kolm 2011; Santini et al. 2019).

Here, we explore these hypotheses to understand the role of brain size on mammalian population densities. Because brain mass can influence energy requirements and cognitive abilities, we expect brain mass to have a direct effect on population density when also considering the effects of body mass and diet composition. If brain size primarily reflects energy requirements, larger brains should lead to lower population densities. However, if the benefits provided by larger brains 
in the form of greater ability to exploit available resources compensate the increased energy requirements, larger-brained species should occur at higher population densities. We test these predictions using Phylogenetic Path Analysis (PPA, von Hardenberg \& Gonzalez-Voyer 2013; Gonzalez-Voyer \& von Hardenberg 2014). This method tests multivariate hypotheses (Shipley 2000) simultaneously solving multiple, related equations that represent direct and indirect relationships among traits, and accounts for potential issues of non-independence in trait data due to shared ancestry (Felsenstein 1985; Martins \& Hansen 1997). Solving multiple, related equations was key in our study because the tested determinants of population density also influence each other. Brain mass has a strong allometric relationship with body mass (Striedter 2005), and larger brain sizes have been linked to diet; for example, in primates those with more frugivorous diets have relatively larger brains (DeCasien, Williams \& Higham 2017). Diet is also linked to body mass with more carnivorous or folivore mammals being larger than those that feed on fruits, nectar, and seeds (Pineda-Munoz, Evans \& Alroy 2016). Considering these relationships, we proposed and compared twelve models (hypotheses) representing different related equations linking diet composition (focusing on percentage of animal items - carnivorous diets, and percentage of specialized plant items: fruit, nectar, and seed), body mass, brain mass, and population density (Fig. 1). We compared model fit and estimated path coefficients to determine which relationships were best supported. 


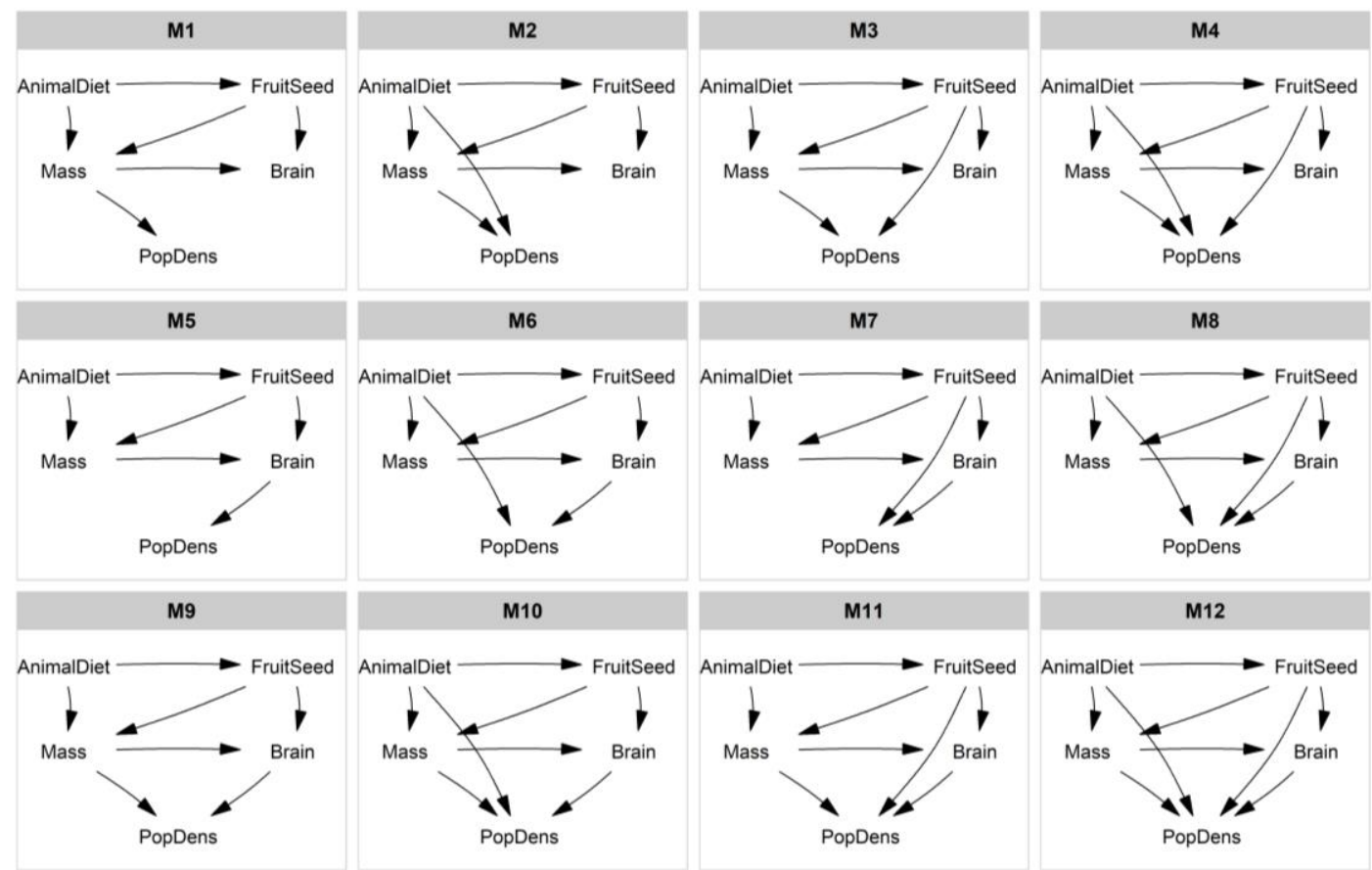

Figure 1. Direct acyclic graphs (DAGs) of the proposed complete path models to explore the effect of diet composition, brain and body mass on population density in non-volant terrestrial mammals. Diet composition is represented by two variables, AnimalDiet: the percentage of the diet represented by all animal items, and FruitSeed: percentage of the diet represented by fruit, nectar, and seed. Relationships between diet and mass reflect those supported for all species analysed together (see methods and Fig. S1 and S2).

\section{Methods}

\section{Data compilation}

We searched the literature, largely focusing on previously published compilations of data from the primary literature, to obtain estimates of population density, body mass, and brain mass for different mammalian species (full dataset available in Figshare repository https://doi.org/10.6084/m9.figshare.12867305.v1). Population density estimates were primarily obtained from two recent compilations of individual study/population records (Novosolov et al. 2017; Santini, Isaac \& Ficetola 2018b). These compilations often included multiple records per species from which we calculated mean densities per species after removing values representing 
introduced and re-introduced populations and duplicates (identical values assumed to represent the same original data). Data gaps were then filled with species-average estimates of population density from other published compilations (details in Supplementary Information). Body and brain mass estimates were compiled from available species-level averages in which individual measurements and sample sizes were rarely reported. We calculated the median value from the available entries after removing identical values, i.e., duplicates (dataset including all individual records available in the Figshare repository https://doi.org/10.6084/m9.figshare.12867305.v1). For many species we had a single estimate of body and brain mass (157 and 208 respectively). For the remaining species the mean number of records was 6.7, with a range from 2-98 for body mass and 7.6 records (range 2103) for brain mass. For species with multiple estimates we calculated variation among available estimates as the percentage difference for each record to the calculated species median: (record value-median)*100/median. Averaging across species the median percentage difference was $10.8 \%$ for body mass and $4.7 \%$ for brain mass. We used this information during the initial data curation process to detect several errors when compiling sources (corrections are described in the compilation scrips available at the Figshare repository https://doi.org/10.6084/m9.figshare.12867305.v1) and afterwards to complete sensitivity analyses (see below).

Diet composition was defined using semi-quantitative measures that reflect percentages of different item categories as described by Wilman et al. (2014). We focused on two main descriptors of diet representing compositions which have been previously linked to population density, body mass and brain mass. We defined the percentage of the diet made up by animal items (AnimalDiet) adding percentages in Wilman et al.'s (2014) categories: ectotherm vertebrates, endotherm vertebrates, fish, unknown vertebrate, insect and scavenged material. We then defined the percentage specialized plant diets (FruitSeed) adding the percentages of three categories: fruit, nectar, and seed. We also tested alternative descriptors of animal diet based on the percentage of insects, and the sum of vertebrate items (categories: ectotherm vertebrates, endotherm vertebrates, 
fish, unknown vertebrate, and scavenged material - assuming scavenging is mostly of vertebrate remains), as well as two alternative descriptors of plant items based on the percentage in the plant (leaves) category, and the seed category alone. We only included empirical diet data, excluding all values imputed based on genus or family information. To define phylogenetic relationships we used the phylogeny in Hedges, Marin, Suleski, Paymer and Kumar (2015). Datasets and scripts of the analyses are available at the Figshare repository https://doi.org/10.6084/m9.figshare.12867305.v1.

\section{Data analyses}

We proposed and tested several possible models describing different direct and indirect relationships among variables using phylogenetic path analysis (von Hardenberg \& GonzalezVoyer 2013). To limit the number of models to compare, we first defined the relationships between diet composition (variables AnimalDiet and FruitSeed), body and brain mass (Fig. S1, Table S1). All tested diet-mass models included a direct link between the two diet variables because these represent percentages of the same total and thus are by definition linked, and a direct effect of body mass on brain mass to reflect their strong allometric relationship. We used the best supported dietmass model as the basis to explore a series of complete models linking diet and mass to population density (Fig. 1). In PPA the links must be directional, in the main results we assumed that the directionality in diet was from AnimalDiet to FruitSeed. The supplementary materials show results were qualitatively the same assuming the alternative direction (Table S2).

All models were fitted and compared using phylogenetic path analysis as implemented in the package 'phylopath' (van der Bijl 2017) in R3.6.1 (R Development Core Team 2019). Prior to analyses we $\log _{10}$-transformed body mass, brain mass and population density, and then scaled all variables (subtracting the mean and dividing by the standard deviation). Because variables are scaled, path coefficients are directly comparable in magnitude. We compared models using the C statistic information criterion CIC (von Hardenberg \& Gonzalez-Voyer 2013). Model averaging was used when possible to incorporate uncertainty in model selection, but defining the complete 
model required a single diet-mass model (a unique set of links). We defined the best supported dietmass model as the one with lowest CIC if a single model was supported (single model with $\Delta \mathrm{CIC}<2$ ). When multiple models were supported we considered the simplest (fewer links) supported model with proposed links between variables having 95\% confidence intervals not overlapping with zero. For complete models linking diet and mass to population density, if several models were supported $(\Delta \mathrm{CIC}<2)$ we used conditional model averaging to calculate path coefficients and their $95 \%$ confidence intervals. We averaged across models that included direct links, met conditional independences, and had $\Delta \mathrm{CIC}<2$ using the function 'average' from the phylopath package. When a single model was supported we calculated path coefficients and bootstrapped $95 \%$ confidence intervals (based on 5000 replicates) using the package 'phylolm' (Tung Ho \& Ané 2014). We analysed all available data combined (all-data model), and separate analyses to evaluate group-specific patterns for taxonomic orders with data for at least 80 species (Primates, Rodentia, Cetartiodactyla, and Carnivora) and for marsupials (Infraclass Metatheria, including species from the taxonomic orders Dasyuromorphia, Didelphimorphia, Diprotodontia and Peramelemorphia). Because we only included terrestrial species to avoid confusion, hereafter we refer to Cetartiodactyla by the former order name Artiodactyla.

Comparative analyses have traditionally explored the role of brain mass using residuals obtained from a log-log brain to body mass regression to capture "relative brain effects", even though, the use of residuals leads to biased parameter estimates (García-Berthou 2001; Freckleton 2002). For comparison we present a supporting analysis based on brain residuals and Phylogenetic Generalized Least Squares (Supplementary Information: Supplementary analyses).

\section{Results}

Representation of extant mammalian diversity in the dataset

We found trait and phylogenetic data for 656 terrestrial non-volant mammals. As expected data were not available for all species, nor did the available data represent a random subset of the 
mammalian diversity, which is a common issue in comparative studies (González-Suárez, Lucas \& Revilla 2012). While the data are not a random sample of all mammals, the dataset included species from 20 taxonomic orders and 92 families (Fig. 2), which spanned several orders of magnitude in body mass, brain mass and population density, and also represented the entire range of diet categories (Figs. S2-S4). There were varying levels of correlation among variables, with body mass and brain mass being most strongly correlated, as expected (Fig S5).
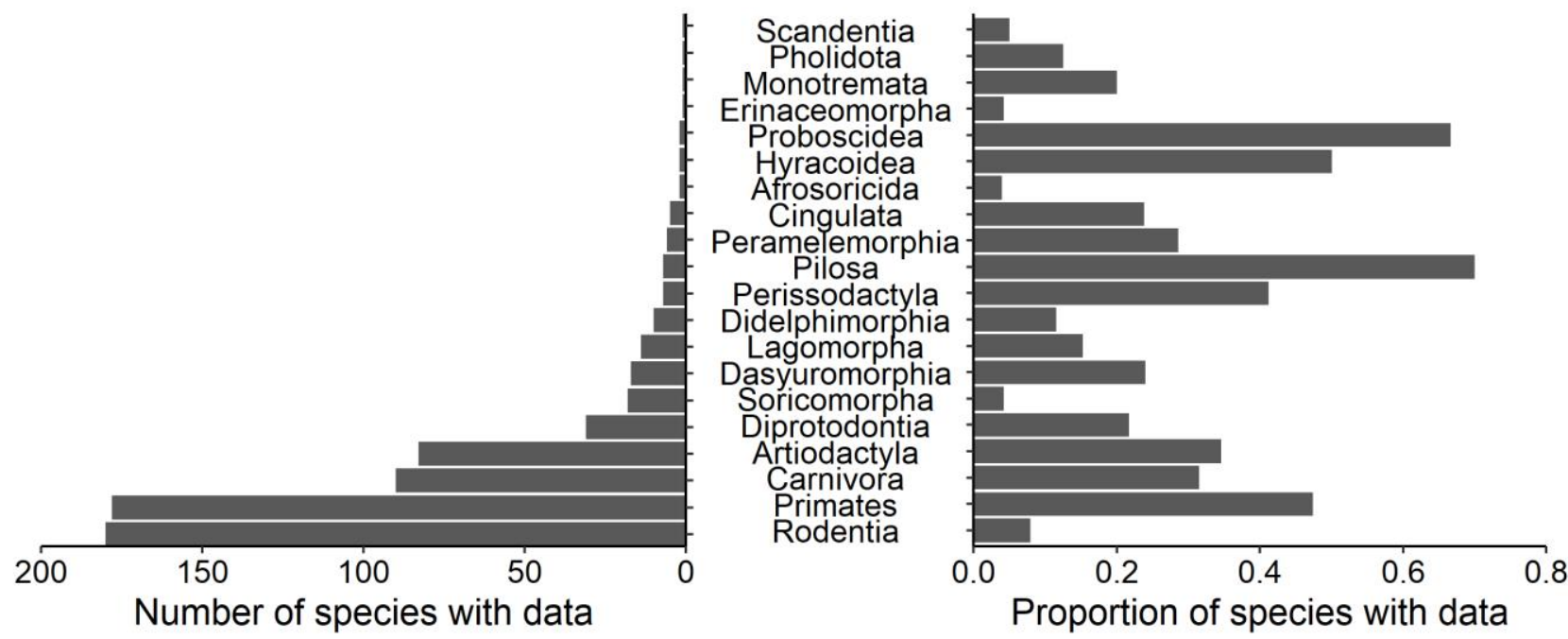

Figure 2. Data availability as total number of species per order and the proportion of species in each order included in the analysed dataset $(\mathrm{N}=656$ species). The comparison shows that representation varied with some species-poor orders like Proboscidea well-represented, but relatively poor coverage of species-rich orders like Rodentia.

\section{Relationships between brain mass, body mass and diet categories}

The best supported model describing relationships between brain, body mass and diet for all species included direct effects of AnimalDiet and FruitSeed (diet variables) on body mass and a direct effect of the percentage of the diet composed by fruits, nectar, and seeds (FruitSeed) on brain mass (Fig S6; Table S1). Alternative diet variables supported the same model structure (Table S1). When considering separate species groups (Primates, Rodentia, Carnivora, Artiodactyla and marsupials) we found varying relationships between diet and brain mass among groups, with overall consistent results when exploring alternative diet variables (Table S3-S7, Fig. S6). 


\section{Brain mass and population density}

The single best-supported complete model for all species (M12, Fig. 1) included direct links from both diet variables, brain mass, and body mass to population density (Table 1). Path coefficient estimates (Fig. 3) showed that lower population densities occur in species with larger brain mass and those that consume diets with higher percentages of animal items or higher percentages of fruits, nectar, and seeds. A direct link between body mass and population density was included in the supported model with a negative path coefficient, but $95 \%$ confidence intervals slightly overlapped zero (best estimate $=-0.29,95 \%$ CI: $-0.600,0.015$ )

Analyses for separate groups showed group-specific idiosyncrasies. We found that primates and carnivorans with larger brains live at lower densities (Fig. 3), but the effect was not clear for rodents, ungulates (order Artiodactyla) and marsupials. Similarly, carnivorans and rodents with larger body mass live at lower population densities, but for primates, ungulates or marsupials the effect was not clear. Unclear effects occurred when one or more of the supported models included the direct link between brain mass or body mass and population density, but the bootstrapped estimates of the $95 \%$ confidence intervals of those path coefficients overlapped zero. In carnivorans both body and brain mass were linked to population density, but contrary to the results from all species in which the only supported model included both direct links, for this group results reflected model averaging of two supported models in which either body mass or brain mass was associated to population density (Table 1). Diet was associated with density in all groups with negative effects of both AnimalDiet and FruitSeed in rodents and primates, positive effects of FruitSeed on carnivorans and ungulates, and negative effects of AnimalDiet on marsupials. 

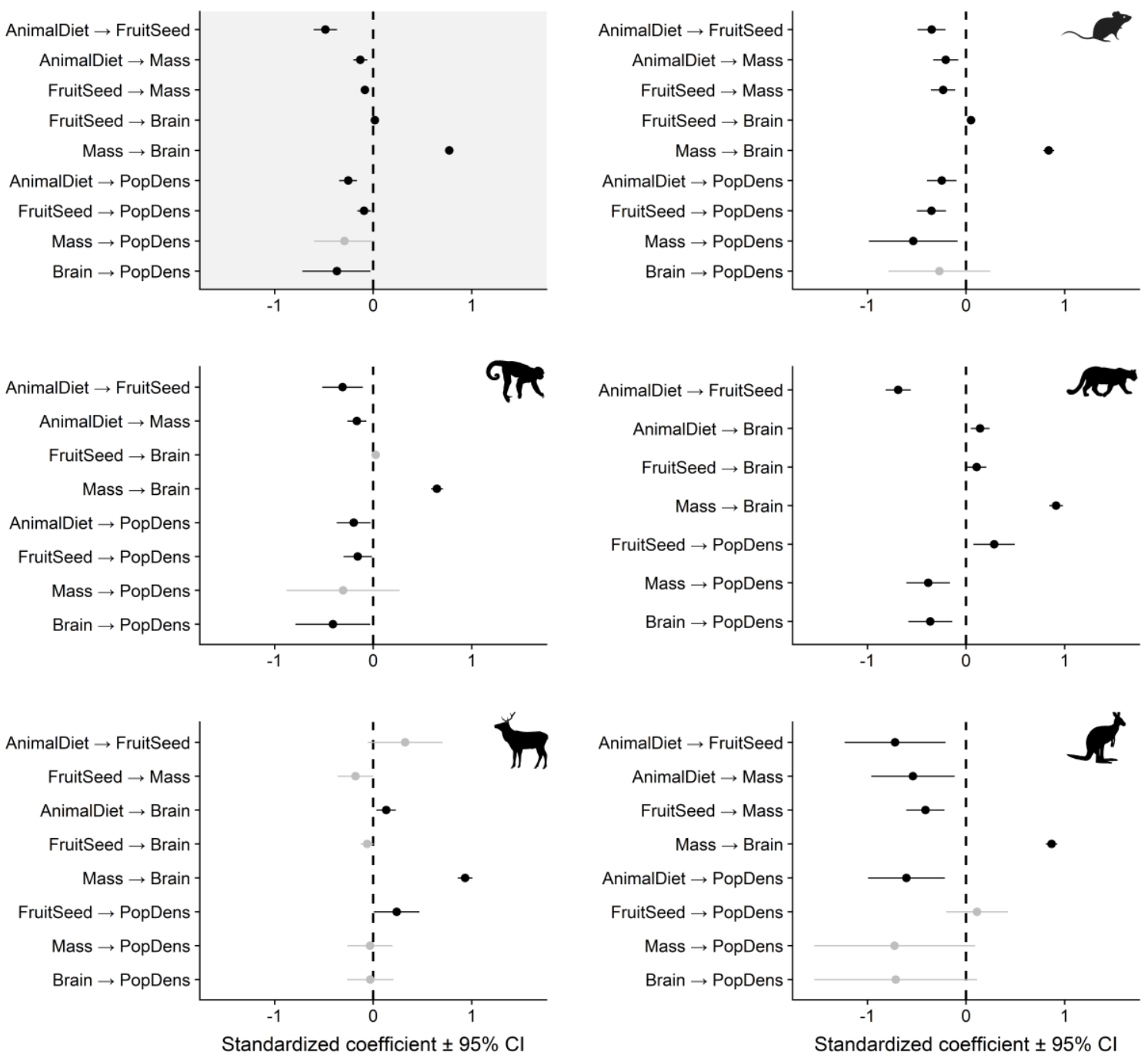

Figure 3. Standardized path coefficients (with 95\% confidence intervals) estimated from the single supported model (for all species, grey background panel) or using model averaging from supported (CIC $<2$ ) models for tested groups (Table 1). Relationships with 95\% confidence intervals not overlapping with zero are shown in black, those overlapping zero are in grey. In some cases 95\% CI were very narrow and lines are not visible. Top left panel with grey background shows results for all 656 non-volant terrestrial mammals, other panels show results for groups tested separately (from top to bottom: rodents, primates, carnivorans, ungulates (Artiodactyla), and marsupials). Figure 1 illustrates the model diagrams. Variables are: AnimalDiet: percentage of the diet composed by animal items; FruitSeed: percentage of the diet composed by fruits, nectar, and seeds, Mass: average adult body mass; Brain: average adult brain mass; PopDens: average population density. 
Table 1. Model comparison for hypothesized relationships between diet, mass and population density for non-volant terrestrial mammalian species (models for all species together and for groups tested separately). Diet is represented by percentage of animal items on the diet (AnimalDiet), and by percentage of the diet composed of fruits, nectar, and seeds (FruitSeed). Supported models used to estimate patch coefficients (Fig. 2) are in bold. We report k: number of independence claims, q: number of parameters, C: Fisher's C statistics, CICc: C-statistic Information Criterion corrected for small sample sizes, $\triangle \mathrm{CICc}$ : difference in CICc from the best-fitting model, w: CICc weights.

\begin{tabular}{|c|c|c|c|c|c|c|c|c|c|}
\hline Model & Diet direct effects & Mass direct effects & $\mathbf{k}$ & $\mathbf{q}$ & $\mathbf{C}$ & $\mathbf{p}$ & CICc & $\triangle \mathrm{CICc}$ & $\mathbf{w}$ \\
\hline \multicolumn{10}{|c|}{ All species $(N=656)$} \\
\hline M12 & AnimalDiet \& FruitSeed & Body \& Brain & 1 & 14 & 3.99 & 0.14 & 32.64 & 0.00 & 0.75 \\
\hline M8 & AnimalDiet \& FruitSeed & Brain & 2 & 13 & 9.35 & 0.05 & 35.92 & 3.28 & 0.15 \\
\hline M4 & AnimalDiet \& FruitSeed & Body & 2 & 13 & 10.50 & 0.03 & 37.07 & 4.43 & 0.08 \\
\hline M10 & AnimalDiet & Body \& Brain & 2 & 13 & 13.77 & 0.01 & 40.34 & 7.70 & 0.02 \\
\hline M6 & AnimalDiet & Brain & 3 & 12 & 16.63 & 0.01 & 41.11 & 8.47 & 0.01 \\
\hline
\end{tabular}

Rodentia $(N=180)$

$\begin{array}{lllllllll}\text { M12 AnimalDiet \& FruitSeed } & \text { Body \& Brain } & 1 & 14 & 3.50 & 0.17 & 34.05 & 0.00 & 0.42\end{array}$




\begin{tabular}{|c|c|c|c|c|c|c|c|c|c|}
\hline Model & Diet direct effects & Mass direct effects & $\mathbf{k}$ & $\mathbf{q}$ & $\mathbf{C}$ & $\mathbf{p}$ & CICc & $\triangle \mathrm{CICc}$ & $\mathbf{w}$ \\
\hline M4 & AnimalDiet \& FruitSeed & Body & 2 & 13 & 5.86 & 0.21 & 34.05 & 0.01 & 0.42 \\
\hline M8 & AnimalDiet \& FruitSeed & Brain & 2 & 13 & 7.87 & 0.10 & 36.06 & 2.02 & 0.15 \\
\hline M11 & FruitSeed & Body \& Brain & 2 & 13 & 15.91 & 0.00 & 44.10 & 10.06 & 0.00 \\
\hline M7 & FruitSeed & Brain & 3 & 12 & 18.27 & 0.01 & 44.14 & 10.09 & 0.00 \\
\hline \multicolumn{10}{|c|}{ Primates $(N=178)$} \\
\hline M8 & AnimalDiet \& FruitSeed & Brain & 3 & 12 & 3.32 & 0.77 & 29.21 & 0.00 & 0.40 \\
\hline M12 & AnimalDiet \& FruitSeed & Body \& Brain & 2 & 13 & 2.49 & 0.65 & 30.71 & 1.49 & 0.19 \\
\hline M4 & AnimalDiet \& FruitSeed & Body & 3 & 12 & 4.94 & 0.55 & 30.83 & 1.62 & 0.18 \\
\hline M6 & AnimalDiet & Brain & 4 & 11 & 9.07 & 0.34 & 32.66 & 3.45 & 0.07 \\
\hline M5 & None & Brain & 5 & 10 & 12.04 & 0.28 & 33.36 & 4.15 & 0.05 \\
\hline
\end{tabular}

Carnivora $(N=90)$

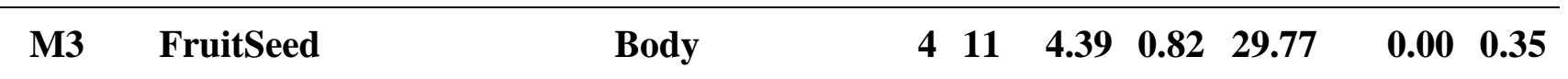




\begin{tabular}{|c|c|c|c|c|c|c|c|c|c|}
\hline Model & Diet direct effects & Mass direct effects & $\mathbf{k}$ & $\mathbf{q}$ & $\mathbf{C}$ & $\mathbf{p}$ & CICc & $\Delta \mathrm{CICc}$ & $\mathbf{w}$ \\
\hline M7 & FruitSeed & Brain & 4 & 11 & 6.10 & 0.64 & 31.48 & 1.71 & 0.15 \\
\hline M4 & AnimalDiet \& FruitSeed & Body & 3 & 12 & 3.73 & 0.71 & 31.78 & 2.01 & 0.13 \\
\hline M2 & AnimalDiet & Body & 4 & 11 & 6.72 & 0.57 & 32.10 & 2.33 & 0.11 \\
\hline M11 & FruitSeed & Body \& Brain & 3 & 12 & 4.05 & 0.67 & 32.11 & 2.33 & 0.11 \\
\hline \multicolumn{10}{|c|}{ Artiodactyla $(N=83)$} \\
\hline M3 & FruitSeed & Body & 3 & 12 & 3.11 & 0.79 & 31.57 & 0.00 & 0.30 \\
\hline M7 & FruitSeed & Brain & 3 & 12 & 3.57 & 0.73 & 32.03 & 0.46 & 0.24 \\
\hline M4 & AnimalDiet \& FruitSeed & Body & 2 & 13 & 2.58 & 0.63 & 33.85 & 2.28 & 0.10 \\
\hline M8 & AnimalDiet \& FruitSeed & Brain & 2 & 13 & 2.72 & 0.61 & 34.00 & 2.43 & 0.09 \\
\hline M11 & FruitSeed & Body \& Brain & 2 & 13 & 3.08 & 0.54 & 34.35 & 2.78 & 0.08 \\
\hline
\end{tabular}

Marsupials $(N=64)$

$\begin{array}{lllllllllll}\text { M6 } & \text { AnimalDiet } & \text { Brain } & & 4 & 11 & 4.33 & 0.83 & 31.41 & 0.00 & 0.28\end{array}$




\begin{tabular}{|c|c|c|c|c|c|c|c|c|c|}
\hline Model & Diet direct effects & Mass direct effects & $\mathbf{k}$ & $\mathbf{q}$ & $\mathbf{C}$ & $\mathbf{p}$ & CICc & $\Delta \mathrm{CICc}$ & $\mathbf{w}$ \\
\hline M2 & AnimalDiet & Body & 4 & 11 & 4.62 & 0.80 & 31.70 & 0.29 & 0.25 \\
\hline M4 & AnimalDiet \& FruitSeed & Body & 3 & 12 & 2.33 & 0.89 & 32.45 & 1.04 & 0.17 \\
\hline M10 & AnimalDiet & Body \& Brain & 3 & 12 & 3.20 & 0.78 & 33.31 & 1.90 & 0.11 \\
\hline M8 & AnimalDiet \& FruitSeed & Brain & 3 & 12 & 3.41 & 0.76 & 33.53 & 2.12 & 0.10 \\
\hline
\end{tabular}




\section{Discussion}

Our results show a negative direct link between average species brain mass and population density in mammals. The link was detected when analysing all 656 species together and clearly supported in separate analyses for primates and carnivorans. For all species and primates supported models included direct links from both body mass and brain mass to population density, although nonsignificant path coefficients indicate a less clear effect of body mass. Support for models that include both direct links suggests brain and body mass estimates could reflect distinct types of drivers or trade-offs. In carnivorans, the two supported models included a direct link from either body mass or brain mass to population density, possibly indicating a more general body size effect on population density where size could be represented by total mass or partial (brain) mass.

The identified links between brain mass and population density are consistent with the hypothesis that higher energetic requirements for species with larger relative brain size lead to lower population densities. Relatively large brains have also been associated with higher cognitive capacity (Reader \& Laland 2002; Sol, Duncan, Blackburn, Cassey \& Lefebvre 2005; BensonAmram, Dantzer, Stricker, Swanson \& Holekamp 2016), which we hypothesized could counterbalance energetic requirements by making individuals better at exploiting resources. While brain size has been linked to species adaptability to novel conditions in mammals (Sol, Bacher, Reader \& Lefebvre 2008; Santini et al. 2019), our results do not support the hypothesis that this benefit compensates the higher energy costs of a larger brain, although this is likely the case for some species such as humans (Navarrete, van Schaik \& Isler 2011). While our findings are consistent with the proposed hypothesis, there could be an alternative, methodological, explanation for a link between brain mass and population density. Brain mass is less variable than body mass during the adult lifespan in mammals and thus, brain mass could act as a more accurate estimate of the species' average adult size rather than an indicator of additional energy requirements. We completed a sensitivity analysis to test this explanation but our findings cannot be explained solely based on brain mass being a more accurate estimate of size (Supplementary Information: 
Supplementary analysis, Table S8). We also tested sensitivity of our results to variation in population density and similarly found results to be robust to potential error in density estimates (Table S8).

Our results could also be consistent with another biological mechanism linking brain size and population density. Larger brains can be associated with more complex social systems (the social brain hypothesis: Dunbar 1998), in these social systems, cooperative group members have the ability to defend much larger territories than the area needed to fulfil the energy requirements of the group (Shultz \& Dunbar 2006). Within particular species, there is evidence of groups with more complex social structures defending larger (per capita) territories (Pasquaretta et al. 2015). Whether territoriality leads to population densities in large-brained social species being lower than expected based only on energy requirements is an intriguing hypothesis that could be explored in future studies. A limitation to such studies is that data on social complexity are sparse and difficult to obtain as potential proxies, like group size, not truly capturing this complexity across mammalian species (e.g., an ungulate herd may be very large but does not have the social cohesion of a smaller primate troop).

As found in previous research (Silva et al. 1997) our analyses link population density with diet. Overall, our results are consistent with a more specialized diet (higher percentages of the diet made up of animal items or specialized plant materials: fruits, nectar, and seeds) being associated with lower population densities for all mammals, rodents, primates, and marsupials. On the other hand, for carnivorans and ungulates, consuming more specialized plant materials (fruits, nectar, and seeds) was associated with higher population densities. Because most carnivorans have animalbased diets, those consuming more fruits, nectar, and seeds (e.g. kinkajou Potos flavus) are effectively less specialized, a result consistent with the general pattern that less specialized diets are associated to larger densities. Similarly, most ungulates are folivores, and those that consume fruits and seeds may be considered less specialized. 
While not the main focus of the study, our results also show interesting relationships between diet composition and brain mass. When analysing all species together we found mammals consuming more fruits, nectar, and seeds had larger brain mass and the same results held for rodents, carnivorans and ungulates. However, in marsupials smaller brains were associated with this specialized diet. Consuming more animal-based diets was associated with larger brains in carnivoran and ungulate species. Contrary to previous work (DeCasien et al. 2017), we did not find a significant link between larger primate brain mass and diets with more fruits, nectar, and seeds, although there was a positive trend (best estimate $=0.025,95 \%$ CI: $-0.003,0.053$ ). Differences may reflect different samples (we included an additional 36 primate species), a different source for diet data, and/or a different analytical approach and phylogeny. Overall, our results suggest that within trophic groups, diets specialized in items that require searching and "capture" (hunting prey or fruit locating and picking) may require more complex behaviours and greater cognitive capacities resulting in larger brain sizes.

Our study shows that mammalian population density is affected by both brain and body mass. Previous research on inter-specific patterns associating mass and population density has focused on total body mass estimates, but our findings suggest that considering both brain and body mass together (or testing them as alternatives) could offer additional information, lead to better predictive models, and reveal groups, such as primates, for which population density may be best explained by brain size. We acknowledge testing both currently presents a challenge because while empirical estimates of body mass are available for nearly 4,900 mammals (Faurby et al. 2018) brain data are available for considerably fewer species ( 1,500 mammals, Tsuboi et al. 2018). Extending the collection of brain mass data would aid future comparative analyses.

Vertebrates with larger brain sizes have been shown to be more successful under certain environments and conditions (Sol et al. 2002; Sol et al. 2008; Amiel et al. 2011; Maklakov et al. 2011; Santini et al. 2019). However, here (and in previous research: Gonzalez-Voyer et al. 2016) we show larger brains also entail ecological costs. Our findings open future avenues of research 
exploring the nuances of how brain size influences population density under different environmental and biotic conditions. Relatively larger brains generally lead to lower population density, but larger brains could allow some species to live at higher densities than expected under challenging or novel conditions. A relatively large brain may provide benefits in the form of more complex social and behavioural strategies that allow species to cope with highly seasonal or unpredictable environments, or to outcompete other species and/or reduce their vulnerability to predators in communities with higher competition and predation pressure. Alternatively, a relatively larger brain might not be associated with higher population densities, but could allow species to persist in a wider array of abiotic and biotic conditions at the cost of lower population densities. These are questions that will help us further understand the role of brain mass in species' ecology and evolution, and how brain size influences a species' abilities to cope with global change.

Acknowledgments: We thank Isabella Capellini for suggesting the social hypothesis under which larger brain size could lead to higher population densities. Credits for silhouettes in figures: Sarah Werning for Cebus albifrons (White-fronted capuchin), Daniel Jaron for Mus musculus (house mouse), Steven Traver for Cervus elaphus (red deer), "An Ignorant Atheist” for Puma concolor (cougar), Cathy for Macropus rufus (red kangaroo). All silhouettes were downloaded from PhyloPic (http://phylopic.org/) and represent species in our dataset.

Author contributions: MGS and AGV conceived the study, all authors designed it and interpreted results, MGS and LS compiled the data, MGS completed all analyses and lead the writing, with contributions from all authors.

\section{Data availability statement}

All data and R scripts (for data compilation and data analyses) are available on the Figshare repository https://doi.org/10.6084/m9.figshare.12867305.v1 


\section{Literature cited}

Amiel, J.J., Tingley, R. \& Shine, R. (2011) Smart moves: effects of relative brain size on establishment success of invasive amphibians and reptiles. PLoS ONE, 6, e18277.

Anderson, K.J. \& Jetz, W. (2005) The broad-scale ecology of energy expenditure of endotherms. Ecology Letters, 8, 310-318.

Barton, R.A. \& Capellini, I. (2011) Maternal investment, life histories, and the costs of brain growth in mammals. Proceedings of the National Academy of Sciences of the United States of America, 108, 6169-6174.

Benson-Amram, S., Dantzer, B., Stricker, G., Swanson, E.M. \& Holekamp, K.E. (2016) Brain size predicts problem-solving ability in mammalian carnivores. Proceedings of the National Academy of Sciences, 113, 2532-2537.

Blackburn, T.M. \& Gaston, K.J. (1999) The relationship between animal abundance and body size: a review of the mechanisms. Advances in ecological research, 28, 181-210.

Brown, J.H. (1995) Macroecology. University of Chicago Press.

Damuth, J. (1981) Population density and body size in mammals. Nature, 290, 699.

Damuth, J. (2007) A macroevolutionary explanation for energy equivalence in the scaling of body size and population density. The American Naturalist, 169, 621-631.

DeCasien, A.R., Williams, S.A. \& Higham, J.P. (2017) Primate brain size is predicted by diet but not sociality. Nat Ecol Evol, 1, 112.

Dobson, A., Tilman, D. \& Holt, R.D. (2020) Unsolved problems in Ecology. Princeton University Press.

Dunbar, R.I. (1998) The social brain hypothesis. Evolutionary Anthropology: Issues, News, and Reviews: Issues, News, and Reviews, 6, 178-190. 
Faurby, S., Davis, M., Pedersen, R.Ø., Schowanek, S.D., Antonelli1, A. \& Svenning, J.-C. (2018) PHYLACINE 1.2: The Phylogenetic Atlas of Mammal Macroecology. Ecology, 99, 26262626.

Felsenstein, J. (1985) Phylogenies and the comparative method. American Naturalist, 125, 1-15.

Freckleton, R.P. (2002) On the misuse of residuals in ecology: regression of residuals vs. multiple regression. Journal of Animal Ecology, 71, 542-545.

García-Berthou, E. (2001) On the misuse of residuals in ecology: testing regression residuals vs. the analysis of covariance. Journal of Animal Ecology, 70, 708-711.

González-Suárez, M., Lucas, P.M. \& Revilla, E. (2012) Biases in comparative analyses of extinction risk: mind the gap. Journal of Animal Ecology, 81, 1211-1222.

Gonzalez-Voyer, A., González-Suárez, M., Vilà, C. \& Revilla, E. (2016) Large brain size indirectly increases vulnerability to extinction in mammals. Evolution, 70, 1364-1375.

Gonzalez-Voyer, A. \& von Hardenberg, A. (2014) Introduction to phylogenetic path analysis. Modern phylogenetic comparative methods and their application in evolutionary biology (ed. L.Z. Garamszegi), pp. 201-229. Springer-Verlag, Berlin.

Hedges, S.B., Marin, J., Suleski, M., Paymer, M. \& Kumar, S. (2015) Tree of life reveals clock-like speciation and diversification. Molecular biology and evolution, msv037.

Herculano-Houzel, S. (2011) Scaling of brain metabolism with a fixed energy budget per neuron: implications for neuronal activity, plasticity and evolution. PLoS ONE, 6, e17514.

Hofman, M.A. (1983) Energy metabolism, brain size and longevity in mammals. The Quarterly Review of Biology, 58, 495-512.

Isaac, N.J., Storch, D. \& Carbone, C. (2011) Taxonomic variation in size-density relationships challenges the notion of energy equivalence. Biology Letters, 7, 615-618.

Isaac, N.J., Storch, D. \& Carbone, C. (2013) The paradox of energy equivalence. Global Ecology and Biogeography, 22, 1-5. 
Isler, K. \& van Schaik, C. (2009) The expensive brain: a framework for explaining evolutionary changes in brain size. Journal of Human Evolution, 57, 392-400.

Isler, K. \& van Schaik, C.P. (2006) Metabolic costs of brain size evolution. Biology Letters, 2, 557560.

Karbowski, J. (2007) Global and regional brain metabolic scaling and its functional consequences. BMC Biology, 5, 18.

Maklakov, A., Immler, S., Gonzalez-Voyer, A., Rönn, J. \& Kolm, N. (2011) Brains and the city: big-brained passerine birds succeed in urban environments. Biology Letters, 7, 730-732.

Martins, E.P. \& Hansen, T.F. (1997) Phylogenies and the comparative method: a general approach to incorporating phylogenetic information into the analysis of interspecific data. American Naturalist, 149, 646-667.

Mink, J.W., Blumenschine, R.J. \& Adams, D.B. (1981) Ratio of central nervous system to body metabolism in vertebrates: its constancy and functional basis. American Journal of Physiology-Regulatory, Integrative and Comparative Physiology, 241, R203-R212.

Nagy, K., Girard, I. \& Brown, T. (1999) Energetics of free-ranging mammals, reptiles, and birds. Annual Review of Nutrition, 19, 247-277.

Navarrete, A., van Schaik, C.P. \& Isler, K. (2011) Energetics and the evolution of human brain size. Nature, 480, 91.

Novosolov, M., Rodda, G.H., North, A.C., Butchart, S.H., Tallowin, O.J., Gainsbury, A.M. \& Meiri, S. (2017) Population density-range size relationship revisited. Global Ecology and Biogeography, 26, 1088-1097.

Pasquaretta, C., Busia, L., Ferrari, C., Bogliani, G., Reale, D. \& Von Hardenberg, A. (2015) Helpers influence on territory use and maintenance in Alpine marmot groups. Behaviour, $152,1391-1412$. 
Pedersen, R.Ø., Faurby, S. \& Svenning, J.-C. (2017) Shallow size-density relations within mammal clades suggest greater intra-guild ecological impact of large-bodied species. Journal of Animal Ecology, 86, 1205-1213.

Pineda-Munoz, S., Evans, A.R. \& Alroy, J. (2016) The relationship between diet and body mass in terrestrial mammals. Paleobiology, 42, 659-669.

R Development Core Team (2019) R: A language and environment for statistical computing. R Foundation for Statistical Computing, Vienna, Austria.

Reader, S.M. \& Laland, K.E. (2002) Social intelligence, innovation, and enhanced brain size in primates. Proceedings of the National Academy of Sciences of the United States of America, 99, 4436-4441.

Santini, L., González-Suárez, M., Russo, D., Gonzalez-Voyer, A., von Hardenberg, A. \& Ancillotto, L. (2019) One strategy does not fit all: determinants of urban adaptation in mammals. Ecology Letters, 22, 365-376.

Santini, L., Isaac, N.J., Maiorano, L., Ficetola, G.F., Huijbregts, M.A., Carbone, C. \& Thuiller, W. (2018a) Global drivers of population density in terrestrial vertebrates. Global Ecology and Biogeography, 27, 968-979.

Santini, L., Isaac, N.J.B. \& Ficetola, G.F. (2018b) TetraDENSITY: A database of population density estimates in terrestrial vertebrates. Global Ecology and Biogeography, 27, 787-791.

Shipley, B. (2000) Cause and correlation in biology: a user's guide to path analysis, structural equations, and causal inference. Cambridge University Press, Cambridge, UK ; New York, NY. USA.

Shultz, S. \& Dunbar, R.I.M. (2006) Both social and ecological factors predict ungulate brain size. Proceedings of the Royal Society B: Biological Sciences, 273, 207-215.

Silva, M., Brimacombe, M. \& Downing, J.A. (2001) Effects of body mass, climate, geography, and census area on population density of terrestrial mammals. Global Ecology and Biogeography, 10, 469-485. 
Silva, M., Brown, J.H. \& Downing, J.A. (1997) Differences in population density and energy use between birds and mammals: a macroecological perspective. Journal of Animal Ecology, 327-340.

Sol, D., Bacher, S., Reader, S.M. \& Lefebvre, L. (2008) Brain size predicts the success of mammal species introduced into novel environments. American Naturalist, 172, S63-S71.

Sol, D., Duncan, R.P., Blackburn, T.M., Cassey, P. \& Lefebvre, L. (2005) Big brains, enhanced cognition, and response of birds to novel environments. Proceedings of the National Academy of Sciences of the United States of America, 102, 5460-5465.

Sol, D., Timmermans, S. \& Lefebvre, L. (2002) Behavioural flexibility and invasion success in birds. Animal Behaviour, 63, 495-502.

Striedter, G.F. (2005) Principles of brain evolution. Sinauer Associates Inc, Sunderland, MA.

Tsuboi, M., van der Bijl, W., Kopperud, B.T., Erritzøe, J., Voje, K.L., Kotrschal, A., Yopak, K.E., Collin, S.P., Iwaniuk, A.N. \& Kolm, N. (2018) Breakdown of brain-body allometry and the encephalization of birds and mammals. Nature Ecology \& Evolution, 2, 1492-1500.

Tung Ho, L.s. \& Ané, C. (2014) A linear-time algorithm for Gaussian and non-Gaussian trait evolution models. Systematic Biology, 63, 397-408.

van der Bijl, W. (2017) phylopath: Perform phylogenetic path analysis. R package version 0.3.1. https://github.com/Ax3man/phylopath.

Visalberghi, E., Fragaszy, D.M. \& Savage-Rumbaugh, S. (1995) Performance in a tool-using task by common chimpanzees (Pan troglodytes), bonobos (Pan paniscus), an orangutan (Pongo pygmaeus), and capuchin monkeys (Cebus apella). journal of Comparative Psychology, 109, 52.

von Hardenberg, A. \& Gonzalez-Voyer, A. (2013) Disentangling evolutionary cause-effect relationships with phylogenetic confirmatory path analysis. Evolution, 67, 378-387.

White, E.P., Ernest, S.K., Kerkhoff, A.J. \& Enquist, B.J. (2007) Relationships between body size and abundance in ecology. Trends Ecol Evol, 22, 323-330. 
Wilman, H., Belmaker, J., Simpson, J., de la Rosa, C., Rivadeneira, M.M. \& Jetz, W. (2014) EltonTraits 1.0: Species-level foraging attributes of the world's birds and mammals. Ecology, 95, 2027-2027. 
Supplementary Information for "The role of brain size on mammalian population densities" Data compilation protocol

All data and R scripts (including those listed here) as available in a Figshare public repository (To add public link upon publication)

\section{Generating brain and body mass estimates ("Brain_data_compilation_published.Rmd")}

We combined all records that provided values for brain and/or body size for mammalian species from several compilations (Mace, Harvey \& Clutton-Brock, 1981; Jeschke \& Strayer, 2006;

Pitnick, Jones \& Wilkinson, 2006; Isler \& Van Schaik, 2009a; Jones et al., 2009; Lemaître, Ramm, Barton \& Stockley, 2009; Weisbecker \& Goswami, 2010; Barton \& Capellini, 2011; Boddy et al., 2012; DeCasien, Williams \& Higham, 2017; Stankowich \& Romero, 2017; Razafindratsima, Yacoby \& Park, 2018; Tsuboi et al., 2018). Body size was always provided as weight, but some sources reported brain size as a volume. Following Isler and van Schaik (2009b), volume estimates (in $\mathrm{ml}$ ) were multiplied by $1.036 \mathrm{~g} / \mathrm{ml}$ (the density of fresh brain tissue) to calculate brain weight. Note that we refer to body and brain mass in the manuscript as these are the most commonly used terms in the literature, strictly speaking body and brain are weight estimates.

A challenge to combine records was that nomenclature was not consistent among datasets and did not always matched the phylogenetic tree we used in our analyses (Hedges, Marin, Suleski, Paymer \& Kumar, 2015). We searched for synonyms of species names using the R libraries taxize (Chamberlain \& Szocs, 2013) and rredlist (Chamberlain, 2018) that query the IUCN, Encyclopedia of Life and ITIS datasets. We searched each name that was not identical to the name in our phylogenetic tree in all three sources (IUCN, Encyclopedia of Life and IT IS) and used this information to define a final accepted name. All cases in which different synonym sources suggested a different synonym were manually checked. We also revised unmatched names and those matched by a single dataset (not found in the others) for accuracy. The entire search protocol and corrections made are reflected in the R script "Brain_data_compilation_published.Rmd". We 
note that the IUCN, Encyclopedia of Life and ITIS datasets are regularly updated and running the searches at different times occasionally resulted in different matches. As noted, we always carefully revised the matches and completed manual edits as needed. Some records could not be matched to any species included in the phylogenetic tree.

The compiled dataset included potential duplicated weight estimates as some recent sources reported data from earlier ones. Because the original source was not always reported it was not always possible to eliminate these duplicates directly. First, to detect potential errors and outliers we considered only distinct brain and body weight values to calculate median and coefficient of variation $(\mathrm{CV})$ estimates per species for both brain and body weight. We then revised all records for species with $\mathrm{CV} \geq 0.60$ for body weight and $\mathrm{CV} \geq 0.30$ for brain mass to detect potential errors. All records were manually investigated with three actions taken: record edited based on the original source if accessible (e.g., correcting typos), record removed if no typo was detected but values were very unlikely or clearly wrong (orders of magnitude higher), or record accepted. The R script "Brain_data_compilation_published.Rmd" provides all details and justifications for all corrections made. Note that because some values were accepted there are species in the dataset that have $\mathrm{CV} \geq 0.60$ for body weight and/or $\mathrm{CV} \geq 0.30$ for brain mass. The final compilation of records (file “Brain_data_compilation_published.csv") includes 6561 records with brain and/or body mass values and list the species name from the original source, the name that matched the phylogenetic tree and the data source. This file includes duplicated values to represent the available data and allow future researchers to use their own criteria for treating duplicates (the section below describes how we considered these).

\section{Generating population density estimates and joining all data}

(“Joining_all_data_published.Rmd")

From the complete compilation of brain and body weight values (file

“Brain_data_compilation_published.csv") we first removed any records not matched to the 
phylogenetic tree phylogeny. We assumed identical mass estimates for the same species represented duplicated records (i.e., the same original measurements) which were removed to generate a dataset including only distinct values of brain and body weight which were used to generate median estimates per species.

Diet data were all obtained from a single source that provide species-level values (Wilman et al., 2014). Binomial species names were matched to names in the brain data compilation (we tested matches to the phylogenetic tree name and the species name from the original brain or body mass data source to maximize matches). If two species in the diet dataset were matched to the same species in the phylogeny, we calculate a mean value.

Population density estimates were obtained from several sources, which reflected both individual population/study records and species averages. We first combined records from two sources that provided estimates from individual studies, and thus often included more than one entry per species (Novosolov et al., 2017; Santini, Isaac \& Ficetola, 2018). We assumed identical values for the same species represented duplicated records (i.e., the same original measurements) which were removed to generate a dataset of unique density estimates. Species names were matched to names in the brain data compilation (we tested matches to the phylogenetic tree name and the species name from the original brain or body mass data source to maximize matches). We then calculated a mean population density value per species. We filled data gaps using additional sources of speciesaverage population density (Jones et al., 2009). There were some inconsistencies in nomenclature that were corrected as detailed in the R script "Joining_all_data_published.Rmd". This script generated the file "Complete_dataset_published.csv" including brain and body mass data for 1349 mammal species (of these 688 species had estimates for all data, including diet and population density estimates. This number includes both terrestrial and marine species, the latter were excluded for our analyses). 


\section{Supplementary tables}

Table S1. Model comparison for hypothesized relationships between diet and mass for 656 mammalian species using different descriptors of diet (described in detail in the main methods). The links between variables are described in Fig. S1, with Fig. S4 showing model coefficients for the main supported model (identified in bold in the table). Only the top 4 models (as ranked by CIC) are shown. k: number of independence claims, q: number of parameters, C: Fisher's C statistic, CICc: C-statistic Information Criterion corrected for small sample sizes, $\triangle \mathrm{CICc}$ : difference in CICc from the best-fitting model, w: CICc weights.

\begin{tabular}{|c|c|c|c|c|c|c|c|c|}
\hline Diet variables & Model & $\mathbf{k}$ & $\mathbf{q}$ & $\mathbf{C}$ & $\mathbf{p}$ & CICc & $\Delta \mathrm{CICc}$ & $\mathbf{w}$ \\
\hline AnimalDiet, FruitSeed & I12 & $\mathbf{1}$ & 9 & 3.99 & 0.14 & 22.27 & 0.00 & 0.90 \\
\hline AnimalDiet, FruitSeed & $\mathrm{I} 4$ & 2 & 8 & 11.61 & 0.02 & 27.83 & 5.57 & 0.06 \\
\hline AnimalDiet, FruitSeed & I8 & 1 & 9 & 10.46 & 0.01 & 28.74 & 6.48 & 0.04 \\
\hline AnimalDiet, FruitSeed & $\mathrm{I} 15$ & 1 & 9 & 16.75 & 0.00 & 35.03 & 12.77 & 0.00 \\
\hline Vertebrate, FruitSeed & I12 & 1 & 9 & 0.27 & 0.87 & 18.55 & 0.00 & 0.77 \\
\hline Vertebrate, FruitSeed & I11 & 2 & 8 & 6.63 & 0.16 & 22.85 & 4.30 & 0.09 \\
\hline Vertebrate, FruitSeed & $\mathrm{I} 15$ & 1 & 9 & 6.36 & 0.04 & 24.64 & 6.09 & 0.04 \\
\hline Vertebrate, FruitSeed & $\mathrm{I} 4$ & 2 & 8 & 8.5 & 0.07 & 24.72 & 6.17 & 0.04 \\
\hline Insectivory, FruitSeed & I12 & $\mathbf{1}$ & 9 & 3.31 & 0.19 & 21.59 & 0.00 & 0.91 \\
\hline Insectivory, FruitSeed & $\mathrm{I} 4$ & 2 & 8 & 11.15 & 0.02 & 27.37 & 5.78 & 0.05 \\
\hline Insectivory, FruitSeed & I8 & 1 & 9 & 9.68 & 0.01 & 27.96 & 6.37 & 0.04 \\
\hline Insectivory, FruitSeed & $\mathrm{I} 14$ & 1 & 9 & 17.68 & 0.00 & 35.95 & 14.36 & 0.00 \\
\hline AnimalDiet, Folivory & I11 & 2 & 8 & 2.75 & 0.60 & 18.97 & 0.00 & 0.40 \\
\hline AnimalDiet, Folivory & $\mathrm{I} 15$ & 1 & 9 & 1.22 & 0.54 & 19.50 & 0.53 & 0.31 \\
\hline AnimalDiet, Folivory & I12 & $\mathbf{1}$ & 9 & 1.53 & 0.47 & 19.81 & 0.84 & 0.27 \\
\hline AnimalDiet, Folivory & $\mathrm{I} 3$ & 3 & 7 & 12.83 & 0.05 & 27.00 & 8.03 & 0.01 \\
\hline AnimalDiet, Granivory & I12 & $\mathbf{1}$ & 9 & 3.62 & 0.16 & 21.90 & 0.00 & 0.97 \\
\hline
\end{tabular}




\begin{tabular}{lcccccccc}
\hline AnimalDiet, Granivory & I4 & 2 & 8 & 14.21 & 0.01 & 30.43 & 8.53 & 0.01 \\
AnimalDiet, Granivory & I8 & 1 & 9 & 13.06 & 0.00 & 31.34 & 9.44 & 0.01 \\
AnimalDiet, Granivory & I15 & 1 & 9 & 15.13 & 0.00 & 33.41 & 11.51 & 0.00 \\
\hline
\end{tabular}


Table S2. Alternative model comparison for hypothesized relationships between diet, mass and population density for mammalian species (all species and for separate groups) in which FruitSeed predicts AnimalDiet. The links between variables are generally described in the model diagrams in Fig. S1 but for these alternative models FruitSeed was the predictor of AnimalDiet (reverse arrow). Model marked in bold were those supported in the main analyses (which are also those supported with this alternative diet relationship). Only the top 5 models (as ranked by CIC) are shown. k: number of independence claims, q: number of parameters, C: Fisher's C statistic, CICc: C-statistic Information Criterion corrected for small sample sizes, $\Delta$ CICc: difference in CICc from the best-fitting model, w: CICc weights.

\begin{tabular}{|c|c|c|c|c|c|c|c|c|c|}
\hline Model & Diet direct effects & Mass direct effects & $\mathbf{k}$ & $\mathbf{q}$ & $\mathbf{C}$ & $\mathbf{p}$ & CICc & $\Delta \mathrm{CICc}$ & $\mathbf{w}$ \\
\hline \multicolumn{10}{|c|}{ All species $(N=656)$} \\
\hline M12 & AnimalDiet \& FruitSeed & Body \& Brain & 1 & 14 & 3.99 & 0.14 & 32.64 & 0.00 & 0.75 \\
\hline M8 & AnimalDiet \& FruitSeed & Brain & 2 & 13 & 9.35 & 0.05 & 35.92 & 3.28 & 0.15 \\
\hline M4 & AnimalDiet \& FruitSeed & Body & 2 & 13 & 10.50 & 0.03 & 37.07 & 4.43 & 0.08 \\
\hline M10 & AnimalDiet & Body \& Brain & 2 & 13 & 13.77 & 0.01 & 40.34 & 7.70 & 0.02 \\
\hline M6 & AnimalDiet & Brain & 3 & 12 & 16.63 & 0.01 & 41.11 & 8.47 & 0.01 \\
\hline
\end{tabular}

Rodentia $(N=180)$ 


\begin{tabular}{|c|c|c|c|c|c|c|c|c|c|}
\hline Model & Diet direct effects & Mass direct effects & $\mathbf{k}$ & $\mathbf{q}$ & $\mathbf{C}$ & $\mathbf{p}$ & CICc & $\Delta \mathrm{CICc}$ & $\mathbf{w}$ \\
\hline M12 & AnimalDiet \& FruitSeed & Body \& Brain & 1 & 14 & 3.50 & 0.17 & 34.05 & 0.00 & 0.42 \\
\hline M4 & AnimalDiet \& FruitSeed & Body & 2 & 13 & 5.86 & 0.21 & 34.05 & 0.01 & 0.42 \\
\hline M8 & AnimalDiet \& FruitSeed & Brain & 2 & 13 & 7.87 & 0.10 & 36.06 & 2.02 & 0.15 \\
\hline M11 & FruitSeed & Body \& Brain & 2 & 13 & 15.91 & 0.00 & 44.10 & 10.06 & 0.00 \\
\hline M7 & FruitSeed & Brain & 3 & 12 & 18.27 & 0.01 & 44.14 & 10.09 & 0.00 \\
\hline \multicolumn{10}{|c|}{ Primates $(N=178)$} \\
\hline M8 & AnimalDiet \& FruitSeed & Brain & 3 & 12 & 3.32 & 0.77 & 29.21 & 0.00 & 0.41 \\
\hline M12 & AnimalDiet \& FruitSeed & Body \& Brain & 2 & 13 & 2.49 & 0.65 & 30.71 & 1.49 & 0.19 \\
\hline M4 & AnimalDiet \& FruitSeed & Body & 3 & 12 & 4.94 & 0.55 & 30.83 & 1.62 & 0.18 \\
\hline M6 & AnimalDiet & Brain & 4 & 11 & 9.07 & 0.34 & 32.66 & 3.45 & 0.07 \\
\hline M5 & None & Brain & 5 & 10 & 12.84 & 0.23 & 34.16 & 4.94 & 0.03 \\
\hline
\end{tabular}

Carnivora $(N=90)$ 


\begin{tabular}{|c|c|c|c|c|c|c|c|c|c|}
\hline Model & Diet direct effects & Mass direct effects & $\mathbf{k}$ & $\mathbf{q}$ & $\mathbf{C}$ & $\mathbf{p}$ & CICc & $\Delta \mathrm{CICc}$ & $\mathbf{w}$ \\
\hline M3 & FruitSeed & Body & 4 & 11 & 3.92 & 0.86 & 29.31 & 0.00 & 0.35 \\
\hline M7 & FruitSeed & Brain & 4 & 11 & 5.63 & 0.69 & 31.02 & 1.71 & 0.15 \\
\hline M4 & AnimalDiet \& FruitSeed & Body & 3 & 12 & 3.26 & 0.78 & 31.31 & 2.01 & 0.13 \\
\hline M2 & AnimalDiet & Body & 4 & 11 & 6.25 & 0.62 & 31.64 & 2.33 & 0.11 \\
\hline M11 & FruitSeed & Body \& Brain & 3 & 12 & 3.59 & 0.73 & 31.64 & 2.33 & 0.11 \\
\hline \multicolumn{10}{|c|}{ Artiodactyla $(N=83)$} \\
\hline M3 & FruitSeed & Body & 3 & 12 & 3.11 & 0.79 & 31.57 & 0.00 & 0.30 \\
\hline M7 & FruitSeed & Brain & 3 & 12 & 3.57 & 0.73 & 32.03 & 0.46 & 0.24 \\
\hline M4 & AnimalDiet \& FruitSeed & Body & 2 & 13 & 2.58 & 0.63 & 33.85 & 2.28 & 0.10 \\
\hline M8 & AnimalDiet \& FruitSeed & Brain & 2 & 13 & 2.72 & 0.61 & 34.00 & 2.43 & 0.09 \\
\hline M11 & FruitSeed & Body \& Brain & 2 & 13 & 3.08 & 0.54 & 34.35 & 2.78 & 0.07 \\
\hline
\end{tabular}

Marsupials $(N=64)$ 


\begin{tabular}{|c|c|c|c|c|c|c|c|c|c|}
\hline Model & Diet direct effects & Mass direct effects & $\mathbf{k}$ & $\mathbf{q}$ & $\mathbf{C}$ & $\mathbf{p}$ & CICc & $\Delta \mathrm{CICc}$ & $\mathbf{w}$ \\
\hline M6 & AnimalDiet & Brain & 4 & 11 & 4.40 & 0.82 & 31.48 & 0.00 & 0.28 \\
\hline M2 & AnimalDiet & Body & 4 & 11 & 4.69 & 0.79 & 31.77 & 0.29 & 0.25 \\
\hline M4 & AnimalDiet \& FruitSeed & Body & 3 & 12 & 2.40 & 0.88 & 32.52 & 1.04 & 0.17 \\
\hline M10 & AnimalDiet & Body \& Brain & 3 & 12 & 3.27 & 0.77 & 33.38 & 1.90 & 0.11 \\
\hline M8 & AnimalDiet \& FruitSeed & Brain & 3 & 12 & 3.48 & 0.75 & 33.60 & 2.12 & 0.10 \\
\hline
\end{tabular}


Table S3. Model comparison for hypothesized relationships between diet and mass for 180

Rodentia species using different descriptors of diet (described in detail in the main methods). The links between variables are described in Fig. S1, with Fig. S4 showing model coefficients for the main supported model (identified in bold in the table). Only the top 4 models (as ranked by CIC) are shown. k: number of independence claims, q: number of parameters, C: Fisher's C statistic, CICc: C-statistic Information Criterion corrected for small sample sizes, $\Delta \mathrm{CICc}$ : difference in CICc from the best-fitting model, w: CICc weights.

\begin{tabular}{lllllllll}
\hline Diet variables & Model & $\mathbf{k}$ & $\mathbf{q}$ & $\mathbf{C}$ & $\mathbf{p}$ & $\mathbf{C I C c}$ & $\Delta \mathbf{C I C}$ & $\mathbf{w}$ \\
\hline AnimalDiet, FruitSeed & $\mathbf{I 1 2}$ & $\mathbf{1}$ & $\mathbf{9}$ & $\mathbf{3 . 5}$ & $\mathbf{0 . 1 7}$ & $\mathbf{2 2 . 5 6}$ & $\mathbf{0 . 0 0}$ & $\mathbf{0 . 8 5}$ \\
AnimalDiet, FruitSeed & $\mathrm{I} 4$ & 2 & 8 & 10.16 & 0.04 & 27.00 & 4.44 & 0.09 \\
AnimalDiet, FruitSeed & I8 & 1 & 9 & 9.53 & 0.01 & 28.59 & 6.03 & 0.04 \\
AnimalDiet, FruitSeed & I15 & 1 & 9 & 12.73 & 0.00 & 31.79 & 9.23 & 0.01 \\
\hline Insectivory, FruitSeed & I12 & $\mathbf{1}$ & $\mathbf{9}$ & $\mathbf{3 . 2 4}$ & $\mathbf{0 . 2 0}$ & $\mathbf{2 2 . 3 0}$ & $\mathbf{0 . 0 0}$ & $\mathbf{0 . 8 2}$ \\
Insectivory, FruitSeed & I4 & 2 & 8 & 9.99 & 0.04 & 26.83 & 4.53 & 0.08 \\
Insectivory, FruitSeed & I8 & 1 & 9 & 9.23 & 0.01 & 28.29 & 5.99 & 0.04 \\
Insectivory, FruitSeed & I15 & 1 & 9 & 9.95 & 0.01 & 29.01 & 6.72 & 0.03 \\
\hline AnimalDiet, Granivory & I12 & $\mathbf{1}$ & $\mathbf{9}$ & $\mathbf{3 . 6 5}$ & $\mathbf{0 . 1 6}$ & $\mathbf{2 2 . 7 1}$ & $\mathbf{0 . 0 0}$ & $\mathbf{0 . 8 3}$ \\
AnimalDiet, Granivory & I4 & 2 & 8 & 9.84 & 0.04 & 26.69 & 3.98 & 0.11 \\
AnimalDiet, Granivory & I8 & 1 & 9 & 9.21 & 0.01 & 28.27 & 5.56 & 0.05 \\
AnimalDiet, Granivory & I15 & 1 & 9 & 16.37 & 0.00 & 35.42 & 12.72 & 0.00 \\
\hline & & & & & & & & \\
\hline
\end{tabular}


Table S4. Model comparison for hypothesized relationships between diet and mass for 178 Primates species using different descriptors of diet (described in detail in the main methods). The links between variables are described in Fig. S1, with Fig. S4 showing model coefficients for the main supported model (identified in bold in the table). Using model I14 to define diet-mass relationships did not qualitatively change the main results. Only the top 4 models (as ranked by CIC) are shown. k: number of independence claims, q: number of parameters, C: Fisher's C statistic, CICc: Cstatistic Information Criterion corrected for small sample sizes, $\triangle \mathrm{CICc}$ : difference in CICc from the best-fitting model, w: CICc weights.

\begin{tabular}{|c|c|c|c|c|c|c|c|c|}
\hline Diet variables & Model & $\mathbf{k}$ & $\mathbf{q}$ & $\mathbf{C}$ & $\mathbf{p}$ & CICc & $\Delta \mathrm{CICc}$ & $\mathbf{w}$ \\
\hline AnimalDiet, FruitSeed & I10 & 2 & 8 & 2.49 & 0.65 & 19.34 & $\mathbf{0 . 0 0}$ & 0.32 \\
\hline AnimalDiet, FruitSeed & $\mathrm{I} 14$ & 1 & 9 & 0.42 & 0.81 & 19.49 & 0.15 & 0.29 \\
\hline AnimalDiet, FruitSeed & $\mathrm{I} 12$ & 1 & 9 & 2.07 & 0.36 & 21.14 & 1.80 & 0.13 \\
\hline AnimalDiet, FruitSeed & I6 & 2 & 8 & 4.48 & 0.34 & 21.34 & 2.00 & 0.12 \\
\hline Insectivory, FruitSeed & $\mathrm{I} 14$ & 1 & 9 & 0.58 & 0.75 & 19.65 & 0.00 & 0.40 \\
\hline Insectivory, FruitSeed & I10 & 2 & 8 & 4.17 & 0.38 & 21.02 & 1.37 & 0.20 \\
\hline Insectivory, FruitSeed & I6 & 2 & 8 & 4.48 & 0.34 & 21.34 & 1.69 & 0.17 \\
\hline Insectivory, FruitSeed & $\mathrm{I} 12$ & 1 & 9 & 3.59 & 0.17 & 22.66 & 3.02 & 0.09 \\
\hline AnimalDiet, Folivory & I14 & 1 & 9 & 0.42 & 0.81 & 19.49 & 0.00 & 0.44 \\
\hline AnimalDiet, Folivory & I6 & 2 & 8 & 4.48 & 0.34 & 21.34 & 1.85 & 0.17 \\
\hline AnimalDiet, Folivory & I10 & 2 & 8 & 5.24 & 0.26 & 22.09 & 2.60 & 0.12 \\
\hline AnimalDiet, Folivory & $\mathrm{I} 2$ & 3 & 7 & 7.72 & 0.26 & 22.38 & 2.89 & 0.10 \\
\hline
\end{tabular}


Table S3. Model comparison for hypothesized relationships between diet and mass for 90 Carnivora species using different descriptors of diet (described in detail in the main methods). The links between variables are described in Fig. S1, with Fig. S4 showing model coefficients for the main supported model (identified in bold in the table). Defining diet-mass relationships with alternative models I5 or I7 did not qualitatively change the main results. Only the top 7 models (as ranked by CIC) are shown. k: number of independence claims, q: number of parameters, C: Fisher's C statistic, CICc: C-statistic Information Criterion corrected for small sample sizes, $\Delta$ CICc: difference in CICc from the best-fitting model, w: CICc weights.

\begin{tabular}{|c|c|c|c|c|c|c|c|c|}
\hline Diet variables & Model & $\mathbf{k}$ & $\mathbf{q}$ & $\mathbf{C}$ & $\mathbf{p}$ & CICc & $\Delta \mathrm{CICc}$ & $\mathbf{w}$ \\
\hline AnimalDiet, FruitSeed & $\mathrm{I} 15$ & 1 & 9 & 0.23 & 0.89 & 20.48 & 0.00 & 0.36 \\
\hline AnimalDiet, FruitSeed & I13 & 2 & 8 & 3.31 & 0.51 & 21.09 & 0.61 & 0.27 \\
\hline AnimalDiet, FruitSeed & $\mathrm{I} 14$ & 1 & 9 & 1.27 & 0.53 & 21.52 & 1.05 & 0.22 \\
\hline AnimalDiet, FruitSeed & $\mathrm{I} 7$ & 2 & 8 & 6.83 & 0.15 & 24.61 & 4.13 & 0.05 \\
\hline AnimalDiet, FruitSeed & I5 & 3 & 7 & 9.91 & 0.13 & 25.27 & 4.80 & 0.03 \\
\hline AnimalDiet, FruitSeed & I6 & 2 & 8 & 7.87 & 0.10 & 25.65 & 5.17 & 0.03 \\
\hline AnimalDiet, FruitSeed & I8 & 1 & 9 & 6.6 & 0.04 & 26.85 & 6.38 & 0.01 \\
\hline Vertebrate, FruitSeed & $\mathrm{I} 3$ & 3 & 7 & 1.62 & 0.95 & 16.98 & 0.00 & 0.19 \\
\hline Vertebrate, FruitSeed & I1 & 4 & 6 & 4.49 & 0.81 & 17.50 & 0.52 & 0.15 \\
\hline Vertebrate, FruitSeed & $\mathrm{I} 2$ & 3 & 7 & 2.74 & 0.84 & 18.10 & 1.12 & 0.11 \\
\hline Vertebrate, FruitSeed & $\mathrm{I} 7$ & 2 & 8 & 0.88 & 0.93 & 18.65 & 1.67 & 0.08 \\
\hline Vertebrate, FruitSeed & $\mathrm{I} 4$ & 2 & 8 & 0.96 & 0.92 & 18.74 & 1.76 & 0.08 \\
\hline Vertebrate, FruitSeed & I5 & 3 & 7 & 3.75 & 0.71 & 19.12 & 2.13 & 0.07 \\
\hline Vertebrate, FruitSeed & $\mathrm{I} 11$ & 2 & 8 & 1.42 & 0.84 & 19.19 & 2.21 & 0.06 \\
\hline Insectivory, FruitSeed & I5 & 3 & 7 & 3.02 & 0.81 & 18.39 & 0.00 & 0.18 \\
\hline Insectivory, FruitSeed & $\mathrm{I} 7$ & 2 & 8 & 0.78 & 0.94 & 18.56 & 0.17 & 0.17 \\
\hline Insectivory, FruitSeed & I1 & 4 & 6 & 6.31 & 0.61 & 19.32 & 0.93 & 0.11 \\
\hline
\end{tabular}


$\begin{array}{llllllllll}\text { Insectivory, FruitSeed } & \text { I3 } & 3 & 7 & 4.07 & 0.67 & 19.44 & 1.04 & 0.11\end{array}$

$\begin{array}{lllllllll}\text { Insectivory, FruitSeed } & \text { I8 } & 1 & 9 & 0.26 & 0.88 & 20.51 & 2.12 & 0.06\end{array}$

$\begin{array}{lllllllllll}\text { Insectivory, FruitSeed } & \text { I13 } & 2 & 8 & 2.76 & 0.60 & 20.54 & 2.15 & 0.06\end{array}$

$\begin{array}{lllllllll}\text { Insectivory, FruitSeed } & \text { I6 } & 2 & 8 & 2.98 & 0.56 & 20.76 & 2.37 & 0.06\end{array}$ 
Table S4. Model comparison for hypothesized relationships between diet and mass for 83

Artiodactyla species using different descriptors of diet (described in detail in the main methods).

The links between variables are described in Fig. S1, with Fig. S4 showing model coefficients for the main supported model (identified in bold in the table). Only the top 4 models (as ranked by

CIC) are shown. k: number of independence claims, q: number of parameters, C: Fisher's C statistic, CICc: C-statistic Information Criterion corrected for small sample sizes, $\Delta \mathrm{CICc}$ : difference in CICc from the best-fitting model, w: CICc weights.

\begin{tabular}{llllllllll}
\hline Diet variables & Model & $\mathbf{k}$ & $\mathbf{q}$ & $\mathbf{C}$ & $\mathbf{p}$ & $\mathbf{C I C c}$ & $\Delta \mathbf{\Delta I C c}$ & $\mathbf{w}$ \\
\hline AnimalDiet, FruitSeed & I15 & $\mathbf{1}$ & $\mathbf{9}$ & $\mathbf{2 . 5 5}$ & $\mathbf{0 . 2 8}$ & $\mathbf{2 3 . 0 1}$ & $\mathbf{0 . 0 0}$ & $\mathbf{0 . 4 2}$ \\
AnimalDiet, FruitSeed & I14 & 1 & 9 & 4.76 & 0.09 & 25.23 & 2.21 & 0.14 \\
AnimalDiet, FruitSeed & I8 & 1 & 9 & 5.01 & 0.08 & 25.48 & 2.46 & 0.12 \\
AnimalDiet, FruitSeed & I7 & 2 & 8 & 7.56 & 0.11 & 25.51 & 2.49 & 0.12 \\
\hline Insectivory, FruitSeed & I15 & $\mathbf{1}$ & $\mathbf{9}$ & $\mathbf{1 . 2 1}$ & $\mathbf{0 . 5 5}$ & $\mathbf{2 1 . 6 8}$ & $\mathbf{0 . 0 0}$ & $\mathbf{0 . 3 6}$ \\
Insectivory, FruitSeed & I7 & 2 & 8 & 4.59 & 0.33 & 22.54 & 0.86 & 0.24 \\
Insectivory, FruitSeed & I8 & 1 & 9 & 3.38 & 0.18 & 23.85 & 2.17 & 0.12 \\
Insectivory, FruitSeed & I13 & 2 & 8 & 6.99 & 0.14 & 24.93 & 3.26 & 0.07 \\
\hline AnimalDiet, Folivory & I15 & $\mathbf{1}$ & $\mathbf{9}$ & $\mathbf{1 . 4 4}$ & $\mathbf{0 . 4 9}$ & $\mathbf{2 1 . 9 1}$ & $\mathbf{0 . 0 0}$ & $\mathbf{0 . 5 2}$ \\
AnimalDiet, Folivory & I7 & 2 & 8 & 6.46 & 0.17 & 24.40 & 2.49 & 0.15 \\
AnimalDiet, Folivory & I14 & 1 & 9 & 4.76 & 0.09 & 25.23 & 3.32 & 0.10 \\
AnimalDiet, Folivory & I8 & 1 & 9 & 5.01 & 0.08 & 25.48 & 3.57 & 0.09 \\
\hline & & & & & & & & \\
\hline
\end{tabular}


Table S7. Model comparison for hypothesized relationships between diet and mass for 64 marsupial species using different descriptors of diet (described in detail in the main methods). Species represent the taxonomic orders Dasyuromorphia, Didelphimorphia, Diprotodontia and Peramelemorphia. The links between variables are described in Fig. S1, with Fig. S4 showing model coefficients for the main supported model (identified in bold in the table). Only the top 4 models (as ranked by CIC) are shown. k: number of independence claims, q: number of parameters, C: Fisher's C statistic, CICc: C-statistic Information Criterion corrected for small sample sizes, $\triangle \mathrm{CICc}$ : difference in CICc from the best-fitting model, w: CICc weights.

\begin{tabular}{|c|c|c|c|c|c|c|c|c|}
\hline Diet variables & Model & $\mathbf{k}$ & $\mathbf{q}$ & $\mathbf{C}$ & $\mathbf{p}$ & CICc & $\Delta \mathrm{CICc}$ & $\mathbf{W}$ \\
\hline AnimalDiet, FruitSeed & I4 & 2 & 8 & 1.75 & 0.78 & 20.37 & 0.00 & 0.50 \\
\hline AnimalDiet, FruitSeed & I8 & 1 & 9 & 0.53 & 0.77 & 21.86 & 1.49 & 0.24 \\
\hline AnimalDiet, FruitSeed & $\mathrm{I} 12$ & 1 & 9 & 0.8 & 0.67 & 22.13 & 1.76 & 0.21 \\
\hline AnimalDiet, FruitSeed & $\mathrm{I} 3$ & 3 & 7 & 10.12 & 0.12 & 26.12 & 5.75 & 0.03 \\
\hline Vertebrate, FruitSeed & I8 & 1 & 9 & 1.94 & 0.38 & 23.27 & 0.00 & 0.65 \\
\hline Vertebrate, FruitSeed & I4 & 2 & 8 & 6.76 & 0.15 & 25.38 & 2.11 & 0.23 \\
\hline Vertebrate, FruitSeed & $\mathrm{I} 12$ & 1 & 9 & 5.6 & 0.06 & 26.93 & 3.66 & 0.10 \\
\hline Vertebrate, FruitSeed & I6 & 2 & 8 & 13.68 & 0.01 & 32.29 & 9.02 & 0.01 \\
\hline Insectivory, FruitSeed & I4 & 2 & 8 & 5.05 & 0.28 & 23.66 & $\mathbf{0 . 0 0}$ & 0.43 \\
\hline Insectivory, FruitSeed & I8 & 1 & 9 & 2.81 & 0.25 & 24.14 & 0.48 & 0.34 \\
\hline Insectivory, FruitSeed & $\mathrm{I} 12$ & 1 & 9 & 3.63 & 0.16 & 24.97 & 1.30 & 0.23 \\
\hline Insectivory, FruitSeed & $\mathrm{I} 2$ & 3 & 7 & 24.82 & 0.00 & 40.82 & 17.16 & 0.00 \\
\hline AnimalDiet, Folivory & I4 & 2 & 8 & 1.75 & 0.78 & 20.37 & 0.00 & 0.35 \\
\hline AnimalDiet, Folivory & $\mathrm{I} 3$ & 3 & 7 & 5.62 & 0.47 & 21.62 & 1.25 & 0.19 \\
\hline AnimalDiet, Folivory & I8 & 1 & 9 & 0.53 & 0.77 & 21.86 & 1.49 & 0.17 \\
\hline AnimalDiet, Folivory & $\mathrm{I} 12$ & 1 & 9 & 1.35 & 0.51 & 22.68 & 2.32 & 0.11 \\
\hline
\end{tabular}




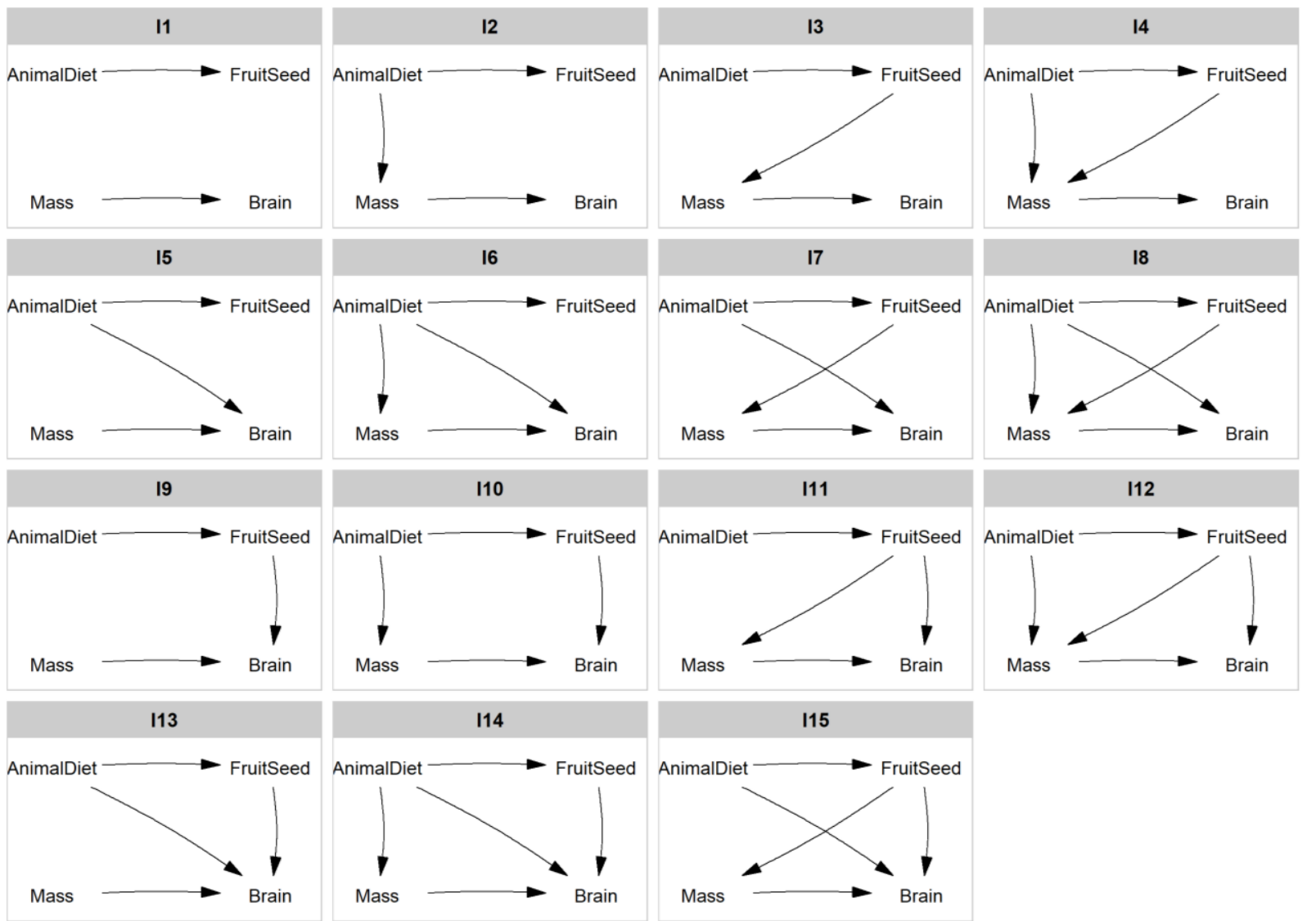

Figure S1. Direct acyclic graphs of the proposed path diet-mass models to explore the effect of diet on body and brain mass. Variables are: AnimalDiet: percentage of the diet composed by animal items; FruitSeed: percentage of the diet composed by fruits, nectar, and seeds, Mass: average adult body mass; Brain: average adult brain mass. 

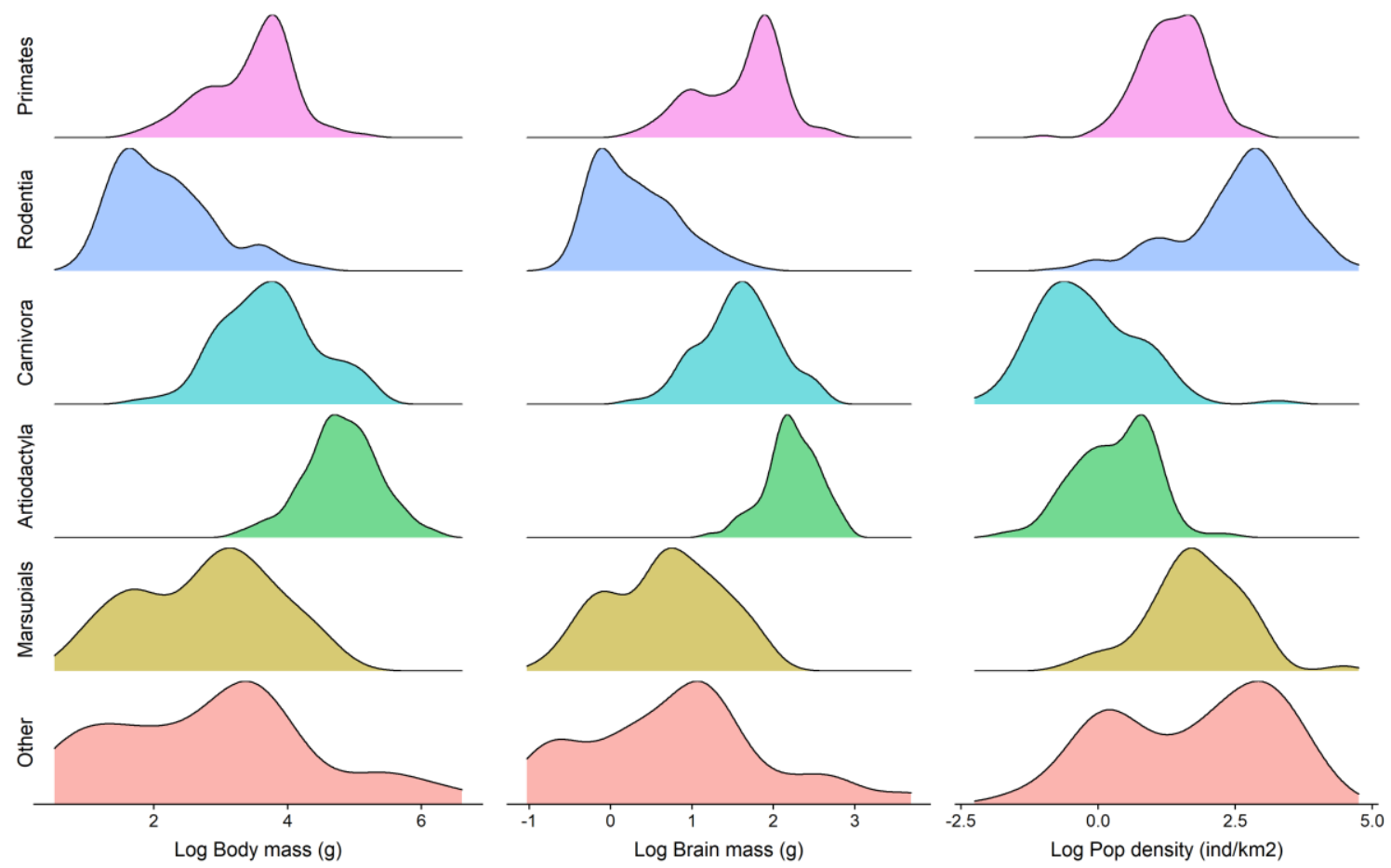

Figure S2. Distribution of body mass, brain mass and population density values for the 656 terrestrial non-volant mammals analysed in this study with groups based on the five groups analysed separately and all other species grouped together.
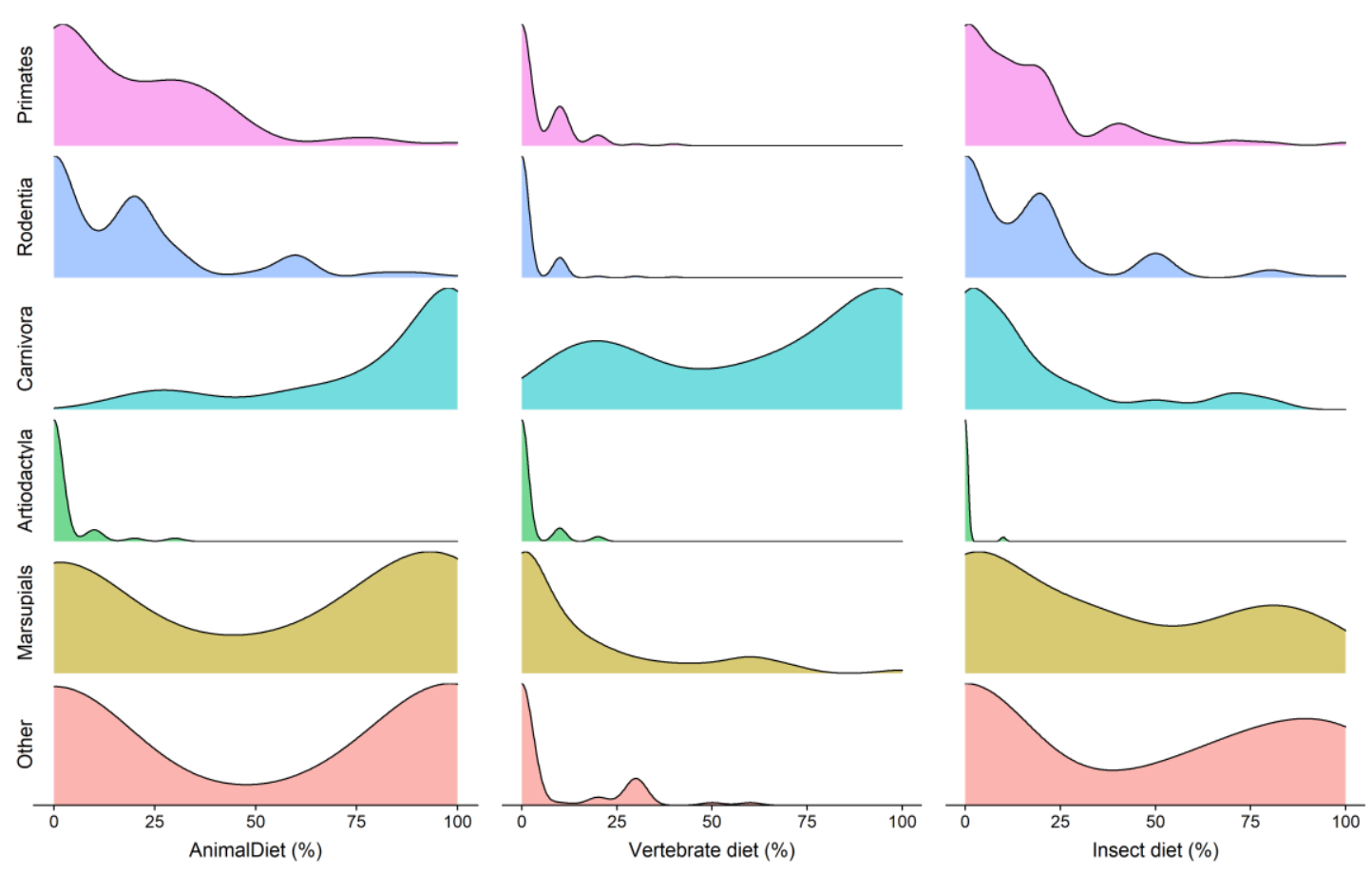

Figure S3. Distribution of animal-based diet categories for the 656 terrestrial non-volant mammals analysed in this study with groups based on the five groups analysed separately and all other species grouped together. 

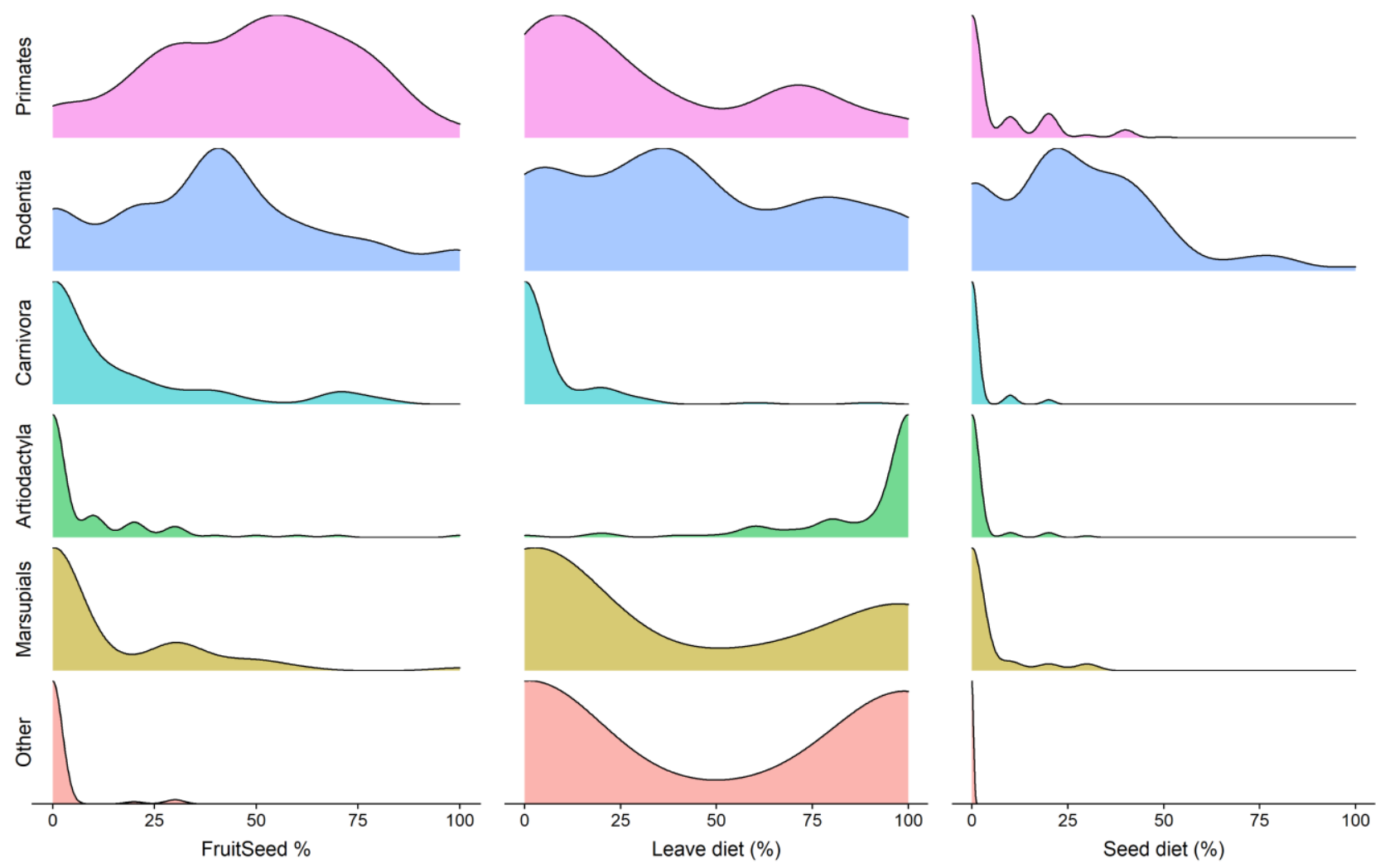

Figure S4. Distribution of plant-based diet categories for the 656 terrestrial non-volant mammals analysed in this study with groups based on the five groups analysed separately and all other species grouped together. 


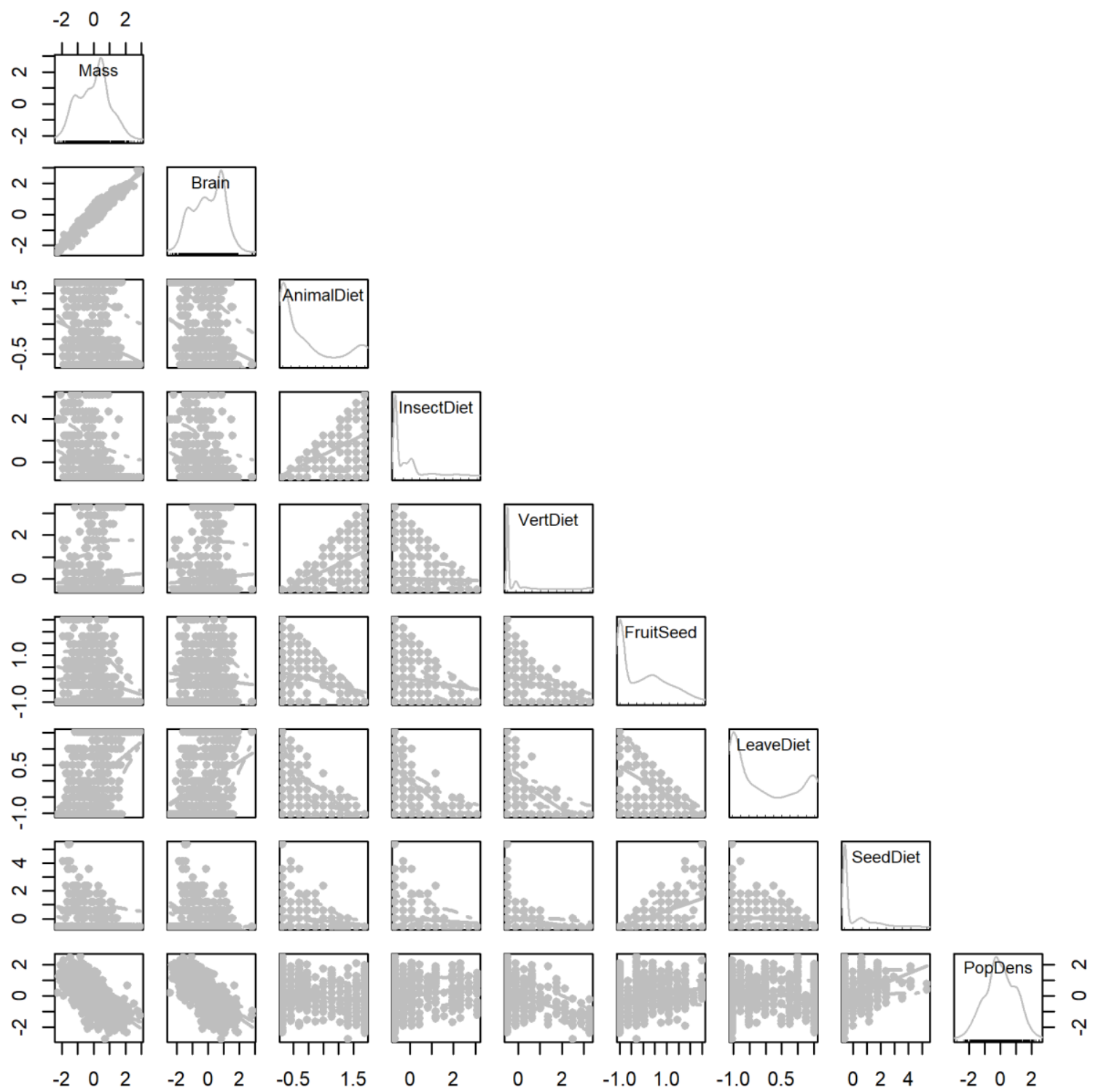

Figure S5. Correlations between trait values for the 656 terrestrial non-volant mammals analysed in this study. Variables are: Mass: average adult body mass; Brain: average adult brain mass; AnimalDiet: percentage of the diet composed by animal items; InsectDiet: percentage of the diet composed by insects; VertDiet: percentage of the diet composed by vertebrates; FruitSeed: percentage of the diet composed by fruits, nectar, and seeds; LeaveDiet: percentage of the diet composed by leaves; SeedDiet: percentage of the diet composed by seeds; PopDens: average population density. 

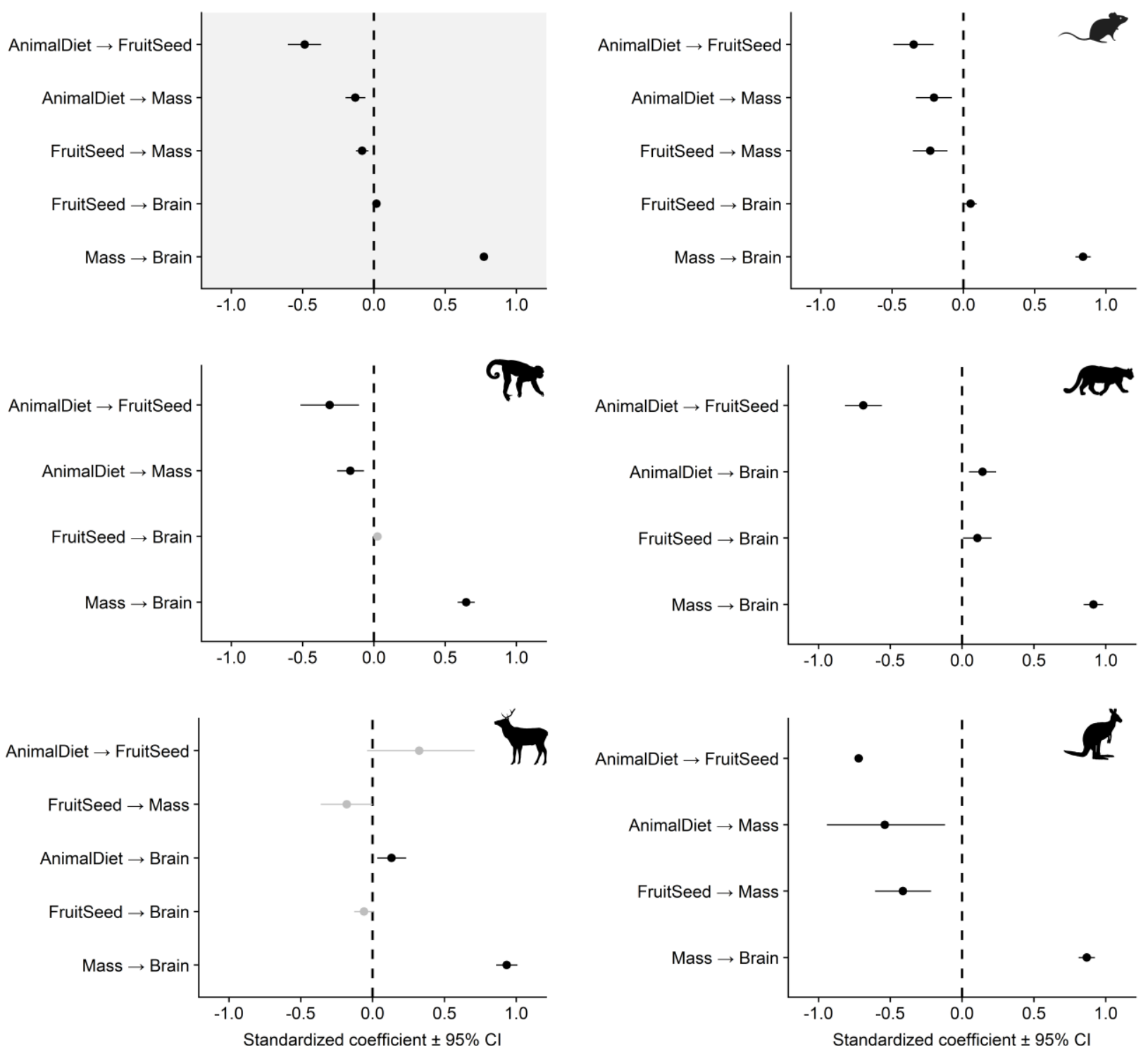

Figure S6. Standardized path coefficients (with 95\% confidence intervals) for the supported dietmass models (Error! Not a valid bookmark self-reference.). Relationships with 95\% confidence intervals not overlapping with zero are shown in black, those overlapping zero are in grey. Top left panel with grey background shows results for all 656 non-volant terrestrial mammal species, other panels show results for groups tested separately (from top to bottom: rodents, primates, carnivora, ungulates (Artiodactyla), and marsupials). Figure S1 illustrates the model diagrams. Variables are: AnimalDiet: percentage of the diet composed by animal items; FruitSeed: percentage of the diet composed by fruits, nectar, and seeds, Mass: average adult body mass; Brain: average adult brain mass. 


\section{Supplementary analyses}

\section{$\underline{\text { Brain residual PLGS }}$}

The role of brain size is sometimes explored using residuals from a log-log brain to body mass regression to capture "relative brain effects". Although using residuals to test highly correlated variables (like body and brain mass) can lead to issues in parameter estimation (Freckleton, 2002), we acknowledge this approach is often used in comparative studies and readers may wonder if our findings would be supported using this traditional approach. We tested if relationships identified in PPA were supported using brain residuals and Phylogenetic Generalized Least Squares (PGLS) models. We fitted PGLS using the phylolm function from the package 'phylolm' with model=lambda estimating the phylogenetic signal using Pagel $\lambda$. We explored alternative evolutionary models including Brownian Motion and Ornstein-Uhlenbeck model, but estimating lambda was better supported (lower AIC). We calculated $\mathrm{R}^{2}$ using the function 'R2' (output $\mathrm{R}_{\text {pred}}$ ) in the rr2 package (Tung Ho \& Ané, 2014; Ives \& Li, 2018). To define standardized brain residuals, we fitted a PGLS regression predicting brain mass as a function of body mass (both $\log _{10^{-}}$ transformed) for all species. Positive residuals indicate species with a larger than expected brain mass given their body mass. We then fitted PGLS regressions to predict population density as a function of body mass, brain residuals and any diet descriptors identified as directly linked to population density in PPA. Body mass were $\log _{10}$-transformed and scaled (subtracting the mean and dividing by the standard deviation). Diet variables were scaled.

PGLS analyses supported PPA results with an effect of relative brain size for all species (Fig. S7) and primates (Table S7). For carnivora, however, relative brain size was not associated with population density when body mass was included. This is consistent with our interpretation that for carnivora average results of the path analyses (which capture two alternative models) indicate the effect of size could be represented by body or brain mass. Indeed, alternative PGLS models including diet and either body mass or brain mass had similar support (AIC within 1 unit). A considerable amount of variance in population density was explained by these models (Table S7). 

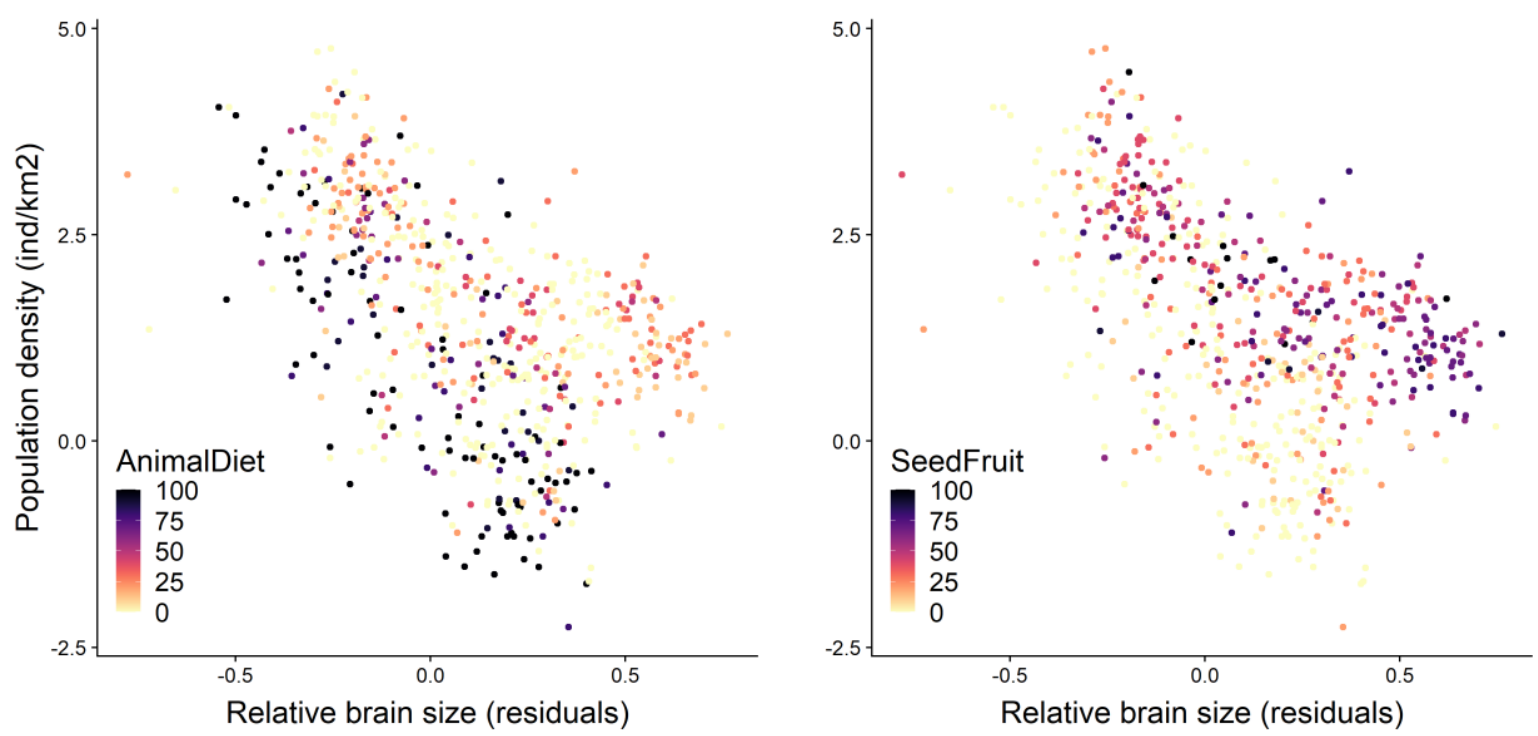

Figure S7. Relationship between relative brain size (residuals from a phylogenetic regression of brain mass and body mass) and population density for 656 mammals. Colour indicates the percentages of diet composed by animal items (left panel) and by fruits, nectar and seeds (right seeds). 
Table S8. Results from PGLS models predicting population density as a function of variables supported in the main PPA analyses. Mass: average adult body mass; Brain residual: residual from a PGLS model predicting $\log _{10}$ average adult brain mass as a function of $\log _{10}$ average adult body mass; AnimalDiet: $\%$ of the diet composed by animal items; FruitSeed: $\%$ of the diet composed by fruits, nectar, and seeds. We report best estimate, its standard error (SE), p-value and the model $\mathrm{R}^{2}$.

\begin{tabular}{lllll}
\hline Variable & Estimate & SE & p-value & $\mathbf{R}^{2}$ \\
\hline All species $(N=656)$ & & & & 0.71 \\
& & & & \\
\hline Mass & -0.76 & 0.069 & $<0.001$ & \\
\hline Brain residual & -0.55 & 0.263 & 0.038 & \\
\hline AnimalDiet & -0.34 & 0.064 & $<0.001$ & \\
& & & & \\
\hline FruitSeed & -0.13 & 0.046 & 0.001 & \\
\hline Rodentia (N= 180) & & & & 0.48 \\
\hline Mass & & & & \\
\hline Brain residual & -0.67 & 0.096 & $<0.001$ & \\
& & & & \\
\hline AnimalDiet & -0.25 & 0.079 & 0.002 & \\
\hline FruitSeed & -0.35 & 0.080 & $<0.001$ &
\end{tabular}

\begin{tabular}{lccll}
\hline Primates $(N=178)$ & & & & 0.20 \\
\hline Mass & -0.23 & 0.075 & 0.003 & \\
& & & & \\
\hline Brain residual & -0.37 & 0.354 & 0.293 & \\
\hline AnimalDiet & -0.12 & 0.056 & 0.035 \\
& & & \\
\hline FruitSeed & -0.09 & 0.046 & 0.044
\end{tabular}

Carnivora $(N=90) \quad 0.37$

\begin{tabular}{lccl}
\hline Mass & -0.36 & 0.107 & 0.001 \\
\hline Brain residual & 0.12 & 0.780 & 0.874 \\
\hline FruitSeed & 0.26 & 0.100 & 0.012
\end{tabular}

Artiodactyla $(N=83) \quad 0.08$




\begin{tabular}{lllll}
\hline Mass & -0.03 & 0.087 & 0.700 & \\
Brain residual & 0.11 & 0.772 & 0.892 & \\
\hline FruitSeed & 0.16 & 0.086 & 0.063 & \\
\hline Marsupials $(N=64)$ & & & & 0.40 \\
\hline Mass & & & & \\
\hline Brain residual & -0.70 & 0.165 & $<0.001$ & \\
\hline AnimalDiet & -0.51 & 0.230 & 0.029 & \\
\hline FruitSeed & 0.10 & 0.141 & 0.485
\end{tabular}

\section{$\underline{\text { Sensitivity analyses }}$}

\section{Brain and body mass}

We generated 1,500 datasets in which estimates of brain mass (500 datasets), body mass (500 datasets), or both (500 datasets) for each species were modified by adding a random value ranging from $-5 \%$ to $+5 \%$ of the empirical estimate (this range was based on the observed median percentage differences in brain mass, see main text). We used each of these 1,500 datasets to fit all complete PPA models for all species together and for the five groups analysed separately using the same approach described in the main methods. We report consistency in model selection in terms of best model frequencies and the total number of supported models, and consistency in path coefficients of brain and mass to population density in terms of frequency of sign and significance of the relationships.

The results from these sensitivity analyses revealed strong consistency in model selection (both in the best models and the number of supported models) for all species and the separate groups (Table S8). Estimates of path coefficients were also generally consistent, revealing strong support for a negative effect of brain mass on population density for all species, primates and carnivorans and more unclear effects for rodents, ungulates and marsupials (Table S8). The negative effect of body mass on rodents and carnivorans was also supported. 


\section{Population density}

To test the robustness of our findings to errors and variation in population density estimates, we also completed sensitivity analyses altering these estimates. We used the same approach as above generating 500 datasets in which for each species empirical population density estimates were modified by adding a random value ranging from $-5 \%$ to $+5 \%$. Results of these analyses reveal strong consistency in model selection and in path coefficient estimates. 
Table S5. Sensitivity analyses results based on simulated datasets in which random error was added to the empirical values of brain mass, body mass, both body and brain mass or population density (500 datasets for each). We report the best supported (lowest CICc) model(s) and number of supported models $(\triangle \mathrm{CICc}<2)$ indicating the percentage of datasets that returned each solution. We also report the brain to population density and body mass to population density path coefficient effect (most frequent sign with percentage of datasets with that solution in parenthesis), as well as the percentage of datasets in which the $95 \% \mathrm{CI}$ of these path coefficients did not overlap with zero (significance). Path coefficients and CI were estimated using model averaging as described in the methods so path coefficient that are not represented in the best model could still have an estimate if other supported models included a direct link.

\begin{tabular}{lllllll}
\hline Added error & Best model & Supported & Brain path coefficient & \multicolumn{2}{l}{ Mass path coefficient } \\
\hline & & & Effect & Significance & Effect & Significance \\
\hline All species & & & & & & \\
\hline Brain & M12(100\%) & $1(99.8 \%)$ & Negative (100\%) & $80.2 \%$ & Negative (100\%) & $19.0 \%$ \\
& & $2(0.2 \%)$ & & & & \\
& & & & & & \\
\hline Mass & M12(100\%) & $1(100 \%)$ & Negative (100\%) & $93.8 \%$ & Negative (100\%) & $5.2 \%$ \\
\hline Brain \& mass & M12(100\%) & $1(99.0 \%)$ & Negative (100\%) & $53.0 \%$ & Negative (100\%) & $86.2 \%$ \\
& & $2(1 \%)$ & & & & \\
\hline Pop density & M12(100\%) & $1(100 \%)$ & Negative (100\%) & $100 \%$ & Negative (100\%) & $0 \%$
\end{tabular}

Rodentia 


\begin{tabular}{|c|c|c|c|c|c|c|}
\hline \multirow[t]{2}{*}{ Brain } & M12 (50.6\%) & $2(60.0 \%)$ & \multirow[t]{2}{*}{ Negative (100\%) } & \multirow[t]{2}{*}{$0.0 \%$} & \multirow[t]{2}{*}{ Negative (100\%) } & \multirow[t]{2}{*}{$97.2 \%$} \\
\hline & M4 (49.4\%) & $3(40.0 \%)$ & & & & \\
\hline \multirow[t]{2}{*}{ Mass } & M12 (54.4\%) & $2(52.0 \%)$ & \multirow[t]{2}{*}{ Negative (100\%) } & \multirow[t]{2}{*}{$0.0 \%$} & \multirow[t]{2}{*}{ Negative (100\%) } & \multirow[t]{2}{*}{$98.0 \%$} \\
\hline & M4 (45.6\%) & $3(48.0 \%)$ & & & & \\
\hline \multirow[t]{3}{*}{ Brain \& mass } & M12 (56.6\%) & $1(0.4 \%)$ & \multirow[t]{3}{*}{ Negative (100\%) } & \multirow[t]{3}{*}{$0.4 \%$} & \multirow[t]{3}{*}{ Negative (100\%) } & \multirow[t]{3}{*}{$91.0 \%$} \\
\hline & M4 (49.2\%) & $2(86.6 \%)$ & & & & \\
\hline & & $3(13.0 \%)$ & & & & \\
\hline \multirow[t]{2}{*}{ Pop density } & M12 (53.2\%) & $2(61.8 \%)$ & \multirow[t]{2}{*}{ Negative (100\%) } & \multirow[t]{2}{*}{$0.0 \%$} & \multirow[t]{2}{*}{ Negative $(100 \%)$} & \multirow[t]{2}{*}{$100.0 \%$} \\
\hline & M4 (46.8\%) & $3(38.2 \%)$ & & & & \\
\hline \multicolumn{7}{|l|}{ Primates } \\
\hline \multirow[t]{4}{*}{ Brain } & M8 (99.4\%) & $1(0.2 \%)$ & \multirow[t]{4}{*}{ Negative $(100 \%)$} & \multirow[t]{4}{*}{$75.4 \%$} & \multirow[t]{4}{*}{ Negative (99.4\%) } & \multirow[t]{4}{*}{$0.0 \%$} \\
\hline & M6 $(0.6 \%)$ & $2(20.2 \%)$ & & & & \\
\hline & & $3(79.3 \%)$ & & & & \\
\hline & & $4(0.2 \%)$ & & & & \\
\hline \multirow[t]{2}{*}{ Mass } & M8 (100\%) & $2(24.6 \%)$ & \multirow[t]{2}{*}{ Negative (100\%) } & \multirow[t]{2}{*}{$81.8 \%$} & \multirow[t]{2}{*}{ Negative (100\%) } & \multirow[t]{2}{*}{$0.0 \%$} \\
\hline & & $3(75.4 \%)$ & & & & \\
\hline Brain \& mass & M8 (99.0\%) & $1(2.8 \%)$ & Negative $(100 \%)$ & $73.0 \%$ & Negative (96.6\%) & $0.0 \%$ \\
\hline
\end{tabular}




\begin{tabular}{|c|c|c|c|c|c|c|}
\hline & M4 (1.0\%) & $\begin{array}{l}2(26.6 \%) \\
3(70.6 \%)\end{array}$ & & & & \\
\hline Pop density & M8 (100\%) & $\begin{array}{l}3(98.8 \%) \\
4(0.4 \%) \\
5(0.8 \%)\end{array}$ & Negative (100\%) & $100 \%$ & Negative (100\%) & $0.0 \%$ \\
\hline Carnivora & & & & & & \\
\hline Brain & M3 (100\%) & $\begin{array}{l}2(98.8 \%) \\
3(1.2 \%)\end{array}$ & Negative (97.4\%) & $96.2 \%$ & Negative (100\%) & $96.2 \%$ \\
\hline Mass & M3 (100\%) & $\begin{array}{l}2(61.4 \%) \\
3(38.4 \%) \\
4(0.2 \%)\end{array}$ & Negative (98.8\%) & $98.4 \%$ & Negative (100\%) & $98.4 \%$ \\
\hline Brain \& mass & M3 (100\%) & $\begin{array}{l}1(0.6 \%) \\
2(60.8 \%) \\
3(37.6 \%) \\
4(1.0 \%)\end{array}$ & Negative (94.6\%) & $93.0 \%$ & Negative (100\%) & $94.2 \%$ \\
\hline Mass & M3 (100\%) & $2(55.0 \%)$ & Negative $(100 \%)$ & $100 \%$ & Negative $(100 \%)$ & $100 \%$ \\
\hline
\end{tabular}




\begin{tabular}{|c|c|c|c|c|c|c|}
\hline \multicolumn{7}{|l|}{ Artiodactyla } \\
\hline \multirow[t]{2}{*}{ Brain } & M3 (72.4\%) & $2(95.5 \%)$ & Negative $(95.0 \%)$ & $0 \%$ & Negative (100\%) & $0 \%$ \\
\hline & M7 (27.6\%) & $3(5.0 \%)$ & & & & \\
\hline \multirow[t]{2}{*}{ Mass } & M3 (85.4\%) & $2(99.8 \%)$ & Negative $(99.8 \%)$ & $0 \%$ & Negative (100\%) & $0 \%$ \\
\hline & M7 (14.6\%) & $3(0.2 \%)$ & & & & \\
\hline \multirow[t]{2}{*}{ Brain \& mass } & M3 (69.0\%) & $2(91.8 \%)$ & Negative $(91.8 \%)$ & $0 \%$ & Negative (100\%) & $0 \%$ \\
\hline & M7 (31.0\%) & $3(8.2 \%)$ & & & & \\
\hline Pop density & M3 (100\%) & $2(100 \%)$ & Negative (100\%) & $0 \%$ & Negative (100\%) & $0 \%$ \\
\hline \multicolumn{7}{|l|}{ Marsupials } \\
\hline \multirow[t]{4}{*}{ Brain } & M6 (60.0\%) & $1(6.2 \%)$ & Negative $(96.8 \%)$ & $43.4 \%$ & Negative $(93.8 \%)$ & $51.4 \%$ \\
\hline & M2 (40.0\%) & $2(7.0 \%)$ & & & & \\
\hline & & $3(33.4 \%)$ & & & & \\
\hline & & $4(53.4 \%)$ & & & & \\
\hline \multirow[t]{2}{*}{ Mass } & M6 (73.2\%) & $1(2.2 \%)$ & Negative (100\%) & $44.6 \%$ & Negative $(97.2 \%)$ & $45.2 \%$ \\
\hline & M2 (26.8\%) & $2(2.6 \%)$ & & & & \\
\hline
\end{tabular}


$3(39.8 \%)$

$4(55.4 \%)$

\begin{tabular}{|c|c|c|c|c|c|c|}
\hline \multirow[t]{5}{*}{ Brain \& mass } & M6 (64.4\%) & $1(12.8 \%)$ & \multirow{5}{*}{\multicolumn{2}{|c|}{ Negative (94.6\%) $54.6 \%$}} & \multirow[t]{5}{*}{ Negative $(87.2 \%)$} & \multirow[t]{5}{*}{$54.0 \%$} \\
\hline & M2 (35.6\%) & $2(13.6 \%)$ & & & & \\
\hline & & $3(33.6 \%)$ & & & & \\
\hline & & $4(39.8 \%)$ & & & & \\
\hline & & $5(0.2 \%)$ & & & & \\
\hline \multirow[t]{4}{*}{ Pop density } & M6 (98.8\%) & $2(2.2 \%)$ & \multirow[t]{4}{*}{ Negative (100\%) } & \multirow[t]{4}{*}{$13.4 \%$} & \multirow[t]{4}{*}{ Negative (100\%) } & \multirow[t]{4}{*}{$13.4 \%$} \\
\hline & M2 (1.2\%) & $3(11.2 \%)$ & & & & \\
\hline & & $4(86.4 \%)$ & & & & \\
\hline & & $5(0.2 \%)$ & & & & \\
\hline
\end{tabular}




\section{References}

Barton, R.A. \& Capellini, I. (2011) Maternal investment, life histories, and the costs of brain growth in mammals. Proceedings of the National Academy of Sciences of the United States of America, 108, 6169-6174.

Boddy, A.M., McGowen, M.R., Sherwood, C.C., Grossman, L.I., Goodman, M. \& Wildman, D.E. (2012) Comparative analysis of encephalization in mammals reveals relaxed constraints on anthropoid primate and cetacean brain scaling. Journal of Evolutionary Biology, 25, 981-94. Chamberlain, S. (2018) rredlist: 'IUCN' Red List Client. R package version 0.5.0. . https://CRAN.Rproject.org/package=rredlist,

Chamberlain, S. \& Szocs, E. (2013) taxize - taxonomic search and retrieval in R. F1000Research, $2,191$.

DeCasien, A.R., Williams, S.A. \& Higham, J.P. (2017) Primate brain size is predicted by diet but not sociality. Nature Ecology and Evolution, 1, 112.

Freckleton, R.P. (2002) On the misuse of residuals in ecology: regression of residuals vs. multiple regression. Journal of Animal Ecology, 71, 542-545.

Hedges, S.B., Marin, J., Suleski, M., Paymer, M. \& Kumar, S. (2015) Tree of life reveals clock-like speciation and diversification. Molecular biology and evolution, msv037.

Isler, K. \& Van Schaik, C.P. (2009a) Why are there so few smart mammals (but so many smart birds)? Biology Letters, 5, 125-129.

Isler, K. \& van Schaik, C. (2009b) Why are there so few smart mammals (but so many smart birds)? Biology Letters, 5, 125-129.

Ives, A. \& Li, D. (2018) rr2: An R package to calculate $\mathrm{R}^{2}$ s for regression models. Journal of Open Source Software, 3, 1028.

Jeschke, J.M. \& Strayer, D.L. (2006) Determinants of vertebrate invasion success in Europe and North America. Global Change Biology, 12, 1608-1619. 
Jones, K.E., Bielby, J., Cardillo, M., Fritz, S.A., O'Dell, J., Orme, C.D.L., . . Michener, W.K. (2009) PanTHERIA: a species-level database of life history, ecology, and geography of extant and recently extinct mammals. Ecology, 90, 2648-2648.

Lemaître, J.F., Ramm, S.A., Barton, R. \& Stockley, P. (2009) Sperm competition and brain size evolution in mammals. Journal of Evolutionary Biology, 22, 2215-2221.

Mace, G.M., Harvey, P.H. \& Clutton - Brock, T. (1981) Brain size and ecology in small mammals. Journal of Zoology, 193, 333-354.

Novosolov, M., Rodda, G.H., North, A.C., Butchart, S.H., Tallowin, O.J., Gainsbury, A.M. \& Meiri, S. (2017) Population density-range size relationship revisited. Global Ecology and Biogeography, 26, 1088-1097.

Pitnick, S., Jones, K.E. \& Wilkinson, G.S. (2006) Mating system and brain size in bats. Proceedings of the Royal Society B: Biological Sciences 273, 719-24.

Razafindratsima, O.H., Yacoby, Y. \& Park, D.S. (2018) MADA: Malagasy Animal trait Data Archive. Ecology, 99, 990.

Santini, L., Isaac, N.J.B. \& Ficetola, G.F. (2018) TetraDENSITY: A database of population density estimates in terrestrial vertebrates. Global Ecology and Biogeography, 27, 787-791.

Stankowich, T. \& Romero, A.N. (2017) The correlated evolution of antipredator defences and brain size in mammals. Proceedings of the Royal Society B: Biological Sciences, 284, 20161857. Tsuboi, M., van der Bijl, W., Kopperud, B.T., Erritzøe, J., Voje, K.L., Kotrschal, A., . . Kolm, N. (2018) Breakdown of brain-body allometry and the encephalization of birds and mammals. Nature Ecology \& Evolution, 2, 1492-1500.

Tung Ho, L.s. \& Ané, C. (2014) A linear-time algorithm for Gaussian and non-Gaussian trait evolution models. Systematic biology, 63, 397-408.

Weisbecker, V. \& Goswami, A. (2010) Brain size, life history, and metabolism at the marsupial/placental dichotomy. Proceedings of the National Academy of Sciences of the United States of America, 107, 16216-16221. 
Wilman, H., Belmaker, J., Simpson, J., de la Rosa, C., Rivadeneira, M.M. \& Jetz, W. (2014)

EltonTraits 1.0: Species-level foraging attributes of the world's birds and mammals. Ecology, 95, 2027-2027. 